\title{
'Candidatus Liberibacter solanacearum' Titers in and Infection Effects on Potato Tuber Chemistry of Promising Germplasm Exhibiting Tolerance to Zebra Chip Disease
}

\author{
C. M. Wallis, J. E. Munyaneza, J. Chen, R. Novy, G. Bester, J. L. Buchman, J. Nordgaard, and P. van Hest
}

First and third authors: U.S. Department of Agriculture-Agriculture Research Services (USDA-ARS), San Joaquin Valley Agricultural Sciences Center, Crop Diseases, Pests and Genetics Research Unit, 9611 S. Riverbend Ave., Parlier, CA 93648; second author: USDA-ARS, Yakima Agricultural Research Laboratory, 5230 Konnowac Pass Road, Wapato, WA 98951; fourth author: USDA-ARS, Small Grains and Potato Germplasm Research Unit, 1693 S. 2700 W., Aberdeen, ID 83210; fifth author: Frito-Lay Inc., 4295 Tenderfoot Road, Rhinelander, WI 54501; sixth and seventh authors: Black Gold Farms, 4320 18th Ave. S., Grand Forks, ND 58201; and eighth author: Bejo Seeds Inc., 1972 Silver Spur Place, Oceano, CA 93445.

Accepted for publication 3 August 2015.

\begin{abstract}
Wallis, C. M., Munyaneza, J. E., Chen, J., Novy, R., Bester, G., Buchman, J. L., Nordgaard, J., and van Hest, P. 2015. 'Candidatus Liberibacter solanacearum' titers in and infection effects on potato tuber chemistry of promising germplasm exhibiting tolerance to zebra chip disease. Phytopathology 105:1573-1584.

Long-term sustainable management of zebra chip (ZC) disease of potato requires development of tolerant or resistant germplasm. To this end, 283 potato varieties and breeding clones were infected with the ZC putative causal agent 'Candidatus Liberibacter solanacearum' (Lso) by potato psyllid vector inoculations in 2010, 2011, 2012, and 2013. Potato germplasm was then examined for development of fresh and fried ZC symptoms. Over multiple years 29 breeding clones exhibited little to no symptoms in freshly cut tuber slices, and five exhibited little to no

To this end, tuber amino acid, sugar, and phenolic levels were compared between noninfected and Lso-infected plants. The five putative tolerant clones had less dramatic shifts in host physiology following Lso infection than the susceptible Atlantic cultivar. This suggested lack of host responses to Lso infection that result in major changes in tuber biochemistry is a potential mechanism of ZC resistance. However, the susceptible Atlantic cultivar did have consistently greater Lso titers compared with two of the tolerant entries, so for these reductions in Lso pathogen progression also might be a factor. Regardless, lack of host responses could still remain one trait that could be used to aid in selection of ZC-resistant potato varieties, as other tolerant lines had infection levels consistent with susceptible Atlantic cultivar. These results also suggest that germplasm derived from relatives of cultivated potato plants are viable sources of $\mathrm{ZC}$ disease resistance.
\end{abstract} symptoms in fried slices. These five presumed tolerant breeding clones were chosen for further screening to determine whether the lack of physiological responses to Lso infection was the cause of observed tolerance.
Additional keywords: carbohydrates, phenolics, plant defense responses, terpenoids, tomato-potato psyllid.
Zebra chip (ZC) disease, putatively caused by 'Candidatus Liberibacter solanacearum' (Lso) (syn. 'Candidatus Liberibacter psyllaurous'), is a serious threat to potato (Solanum tuberosum L.) production in Central America, Western North America, and New Zealand (Crosslin et al. 2012a, 2012b; Liefting et al. 2008; Munyaneza 2012; Munyaneza et al. 2007; Secor and Rivera-Varas 2004; Wen et al. 2009). Potato psyllids (sometimes called tomato-potato psyllids), Bactericera cockerelli (Sulc.) (Hemiptera: Triozidae), vector Lso to potato and other solanaceous crops and are the primary source of ZC spread. As a result, current ZC management involves costly ( $\sim \$ 700 /$ ha) chemical control programs for potato psyllids (Guenthner et al. 2012, Greenway 2014). The continued use of insecticides for psyllid control could result in resistant insect populations that would render chemical-based control ineffective (Prager et al. 2013). Likewise, organic and other nonconventional growers would not be allowed to utilize pesticides. Therefore, longterm sustainable management for $\mathrm{ZC}$ disease requires exploiting natural host resistance to develop ZC-resistant or Lso infectiontolerant varieties (Munyaneza et al. 2011; Munyaneza 2012). These Lso-tolerant varieties could be potentially coupled with traits for

Corresponding author: C. M. Wallis;

E-mail address: christopher.wallis@ars.usda.gov

http://dx.doi.org/10.1094/PHYTO-02-15-0040-R

This article is in the public domain and not copyrightable. It may be freely reprinted with customary crediting of the source. The American Phytopathological Society, 2015 psyllid resistance to provide comprehensive protection from ZC (Butler et al. 2011; Cooper and Bamberg 2014; Diaz-Montano et al. 2014; Levy and Tamborindeguy 2014).

One of the biggest concerns with potatoes infected by $\mathrm{ZC}$ is symptoms that reduce the quality and overall marketability of fresh-market and processing tubers. Symptoms characteristic of ZC include increased oxidation (browning) and brown specking around the vascular tissues of freshly cut tubers and brown striping ('zebra stripes') when tuber slices are fried to make potato chips/crisps (Munyaneza 2012; Secor et al. 2009; Wen et al. 2009). In addition to symptoms in tubers, ZC causes above-ground portions of potato plants to undergo dwarfing, leaf scorch, chlorosis, and collapse (Munyaneza 2012; Secor et al. 2009; Wen et al. 2009).

Also characteristic of ZC is substantial changes in tuber physiology, with tubers from Lso-infected plants having greater levels of phenolic compounds (Alvarado et al. 2012; Navarre et al. 2009; Rashed et al. 2013; Wallis et al. 2012, 2014), reducing sugars (glucose and fructose) (Buchman et al. 2011a, 2012; Rashed et al. 2013; Wallis et al. 2014), defense-associated enzymes (Alvarado et al. 2012, Rashed et al. 2013, Wallis et al. 2012), amino acids (e.g., isoleucine, leucine, serine, tyrosine, and valine) (Navarre et al. 2009; Rashed et al. 2013, Wallis et al. 2012, 2014), and minerals (e.g., phosphorus, potassium, and calcium) (Miles et al. 2009) compared with noninfected tubers. Furthermore, changes in foliar and stem levels of amino acids, sugars, phenolics, and terpenoids may occur when potato hosts are infected with Lso, with potential implications on the expression of $\mathrm{ZC}$ symptoms (Wallis et al. 2015). 
Many of these increases in compound levels could be part of a coordinated defense response to Lso infection (Miles et al. 2010), especially increased phenolic compound levels, which are relatively consistent occurrences in potato tissues following various pathogen infections (Henriquez et al. 2012; Ngadze et al. 2012). It remains unlikely that these defenses are effective at halting Lso growth within susceptible potato hosts because otherwise $\mathrm{ZC}$ would not be a concern. However, some evidence does exists that Lso could be halted somewhat as lower titers might be observed where shifts in host physiology are greatest (Rashed et al. 2013; Wallis et al. 2012, 2014).

It is hypothesized that Lso itself likely does not cause many direct negative effects on its potato hosts, but rather host responses to bacterial presence result in ZC symptom formation (Rashed et al. 2013; Wallis et al. 2012, 2014). To be specific, greater phenolic (especially chlorogenic acid and its isomers) and polyphenol oxidase levels result in increased oxidative browning to occur upon exposure to air, as observed in freshly cut susceptible potato tubers infected with Lso (Mayer 2006). Greater levels of sugars and amino acids would provide more substrates for the Maillard reaction when potato slices are fried, with the result being increased formation of the brown-colored acrylamide (Friedman and Levin 2008; Stadler et al. 2002).

This study summarizes ongoing work to discover Lso infectiontolerant potato varieties. This study proceeded further by examining whether or not lack of host physiology shifts were responsible for putative Lso tolerance. This was done by comparing noninfected and Lso-infected tuber levels of amino acids, sugars, and phenolics of tolerant varieties with the same comparisons done for the susceptible Atlantic variety as a reference. If the lack of a host physiological response is consistent in putatively tolerant varieties, then this trait could be selected for in breeding programs aimed at developing germplasm that is tolerant of Lso infection. Such Lso-tolerant varieties will prove invaluable at countering the threat $\mathrm{ZC}$ poses to potato growers in areas where it is endemic.

\section{MATERIALS AND METHODS}

Germplasm screening. Over a 4-year period (2010 to 2013), 283 potato varieties and advanced breeding lines were planted in the field at the USDA-ARS research farm at Moxee, WA. These potatoes were contained in $1.83 \mathrm{~m}$ by $4.57 \mathrm{~m}$ cages, with each cage enclosing six to eight plants each, as described by Munyaneza et al. (2007) and Buchman et al. (2011a, b). Plants were inoculated with Lso by being exposed to Lso-positive potato psyllid adults (three insects/plant) at tuber initiation stage for 7 days, after which time the insects were eliminated through the use of pesticides and the plants were allowed to develop until harvest (Buchman et al. 2011a, b; Munyaneza et al. 2007). Upon harvest, the tubers were assessed for both fresh and fried ZC symptoms, as well as Lso infection confirmed by polymerase chain reaction (PCR) (Buchman et al. 2011a, b; Munyaneza et al. 2007).

Based on findings from the first 2 years (2010 and 2011), a total of five promising breeding clones were selected for further evaluation in 2012 and 2013. The promising clones were: ARS1 (also known as 00-3115-2) and ARS2 (also known as 00-3115-11or M5 [Jansky et al. 2011]) from the USDA-ARS, Madison, WI and Aberdeen, ID; BS2 (also known as T47022) from Bejo Seeds, Inc., Oceano, CA; and ZC73 and ZC74 from Frito-Lay, Inc., Rhinelander, WI. The ARS breeding clones were bred for resistance to cold-induced sweetening and have been characterized as having $25 \%$ of pedigree derived from wild potato species including Solanum raphanifolium, S. tarijense, and S. chacoense (Jansky et al. 2011). Each of these potato clones had six plants established in each of six cages. The susceptible cultivar Atlantic was established similarly for comparison. The promising clones and the susceptible Atlantic cultivar had half of the cages exposed to Lso-infective psyllids at tuber initiation stage, and the remaining cages were left as controls. At harvest tubers were collected, ZC fresh and fried symptoms were assessed, and Lso infection confirmed by PCR as described by Munyaneza et al. (2007) and Buchman et al. (2011a, b). Subsets of noninfected and Lso-infected tubers for each entry were then shipped to USDAARS, Parlier, CA, for evaluation of tuber physiology as described below.

Biochemical analyses. For 2012, a total of nine Lso-infected and three noninfected tubers for each of the selected putatively tolerant breeding clones and susceptible Atlantic tubers were analyzed further, and for 2013 a total of five noninfected and five Lso-infected tubers were analyzed. Upon arrival to Parlier, CA, both noninfected and Lso-infected tubers from both 2012 and 2013 were sliced at the stolon end and examined for fresh ZC symptoms on a 0 to 3 scale (Rashed et al. 2013). Afterward, two additional small slices were made from each tuber. One slice per tuber was placed in a deep fryer containing corn oil for $3 \mathrm{~min}$ at $340^{\circ} \mathrm{F}\left(171^{\circ} \mathrm{C}\right)$, at which time the potato "chip" was removed and evaluated for fried ZC symptoms on a 0 (normal) to 3 (severe) scale. The other slice per tuber had the skin removed and was mashed using a mortar and pestle. The mashed tissue was then used to make two 0.10 -g aliquots in labeled $1.5-\mathrm{ml}$ microcentrifuge tubes, with the remaining mashed tissue saved for Lso titer analyses. These samples then were placed at $-20^{\circ} \mathrm{C}$ until extractions were performed.

Lso titers were determined using DNA extracted using the DNeasy Plant mini kit (Qiagen, Valencia, CA) and the methods of Wallis et al. (2012) on both Lso-inoculated and noninoculated tubers. In brief, quantitative PCR to determined Lso titers involved $2 \mu \mathrm{l}$ of extracted tissue DNA template, $12.5 \mu \mathrm{l}$ of $2 \times$ iQ SYBR Green supermix (Bio-Rad, Herculus, CA), $0.5 \mu \mathrm{l}$ of both $10 \mu \mathrm{M}$ OA2 and $10 \mu \mathrm{M}$ OI2c primers (Liefting et al. 2008), and $9.5 \mu \mathrm{l}$ of water for a total reaction solution volume of $25 \mu \mathrm{l}$ (Wallis et al. 2012). Ct values were calculated by the DNA Engine Opticon 2 Real-Time PCR Detection System (Bio-Rad) that was used to monitor the reactions, with control Lso template used to convert $\mathrm{Ct}$ values to nanogram template (Wallis et al. 2012).

To calculate amino acid, sugar, and phenolic levels, the methods of Rashed et al. (2013) and Wallis et al. (2008, 2012, 2014) were employed.

Briefly, one of the 0.10 -g aliquots from each sample was twiceextracted overnight at $4^{\circ} \mathrm{C}$ in $0.5 \mathrm{ml}$ of phosphate buffered saline (PBS) solution ( $\mathrm{pH} 7.8$ ) for use in amino acid and sugar analyses, for a total of $1 \mathrm{ml}$ of extract. For amino acid analyses, $100 \mu \mathrm{l}$ of this PBS buffer extract was used with the commercially available kit from Phenomenex (Torrance, CA) developed for analyzing amino acids by gas chromatography. Derivatized amino acid extracts from the kit were analyzed with a Shimadzu (Columbia, MD) GC-2010 gas chromatograph with a flame ionization detector and the kitprovided Zebron AAA column. Amino acid identifications and quantification were made using standard preparations from the Phenomenex amino acid kit and the Shimadzu Lab Solutions software.

Sugar analysis involved injecting $50 \mu \mathrm{l}$ of the PBS buffer extract into a Shimadzu high-performance liquid chromatograph (HPLC) equipped with $300 \mathrm{~mm}$ by $7.8 \mathrm{~mm}$ Supelco C-611 carbohydrate column (Sigma-Aldrich, St. Louis, MO) and a refractive index detector (RID-10 from Shimadzu) (Rashed et al. 2013). Commercial standards of fructose and glucose were obtained from Sigma and used to identify peaks and convert peak areas to gram amounts.

To analyze phenolics, the remaining $0.10 \mathrm{~g}$ tuber tissue aliquot was twice-extracted overnight at $4^{\circ} \mathrm{C}$ in $0.5 \mathrm{ml}$ of methanol (Sigma) each time, for a total of $1 \mathrm{ml}$ of extract. A total of $50 \mu \mathrm{l}$ of methanol extract per sample was injected into a Shimadzu HPLC system equipped with an XR-ODS C18 column (Shimadzu) and a Shimadzu photodiode array detector (set at $280 \mathrm{~nm}$ ) to quantify phenolic compounds (Wallis et al. 2008, 2012). The binary solvent gradient used in the HPLC proceeded from $95 \%$ solvent A (water with $0.2 \%$, vol/vol, acetic acid [Sigma]) to $100 \%$ solvent B (methanol with $0.2 \%$, vol $/ \mathrm{vol}$, acetic acid) and back to reset for the following 
injection over $40 \mathrm{~min}$ (Wallis et al. 2012). The majority of tuber phenolics were identified by Wallis et al. (2012) by matching retention times with commercial standards (from Sigma), matching UV/Vis spectra of peaks, or determining molecular weights by running representative samples on a Shimadzu LCMS-2020 liquid chromatograph-mass spectrometer. Area units of quantified peaks were converted to gram amounts by using standard curves to develop conversion factors for compounds within the same phenolic class and the same UV/Vis spectral profile (Rashed et al. 2013).

Statistical analyses. IBM-SPSS software version 22.0 (IBM, Armonk, NY) was used in all analyses with $\alpha=0.05$. Normality was tested with the SPSS EXPLORE feature by examining residual plots. $t$ tests were used to determine differences in symptoms and Lso titers between noninfected and infected tubers for each entry. Multivariate analyses of variance (MANOVA) of amino acids, sugars, or phenolics was utilized to determine significant differences between noninfected and infected plants for each of the tested entries for both years as well as each year separately. If the MANOVA Pillai's trace statistic was significant, differences in individual compounds were examined using follow-up analyses of variance (ANOVAs). ANOVAs were performed for all individual

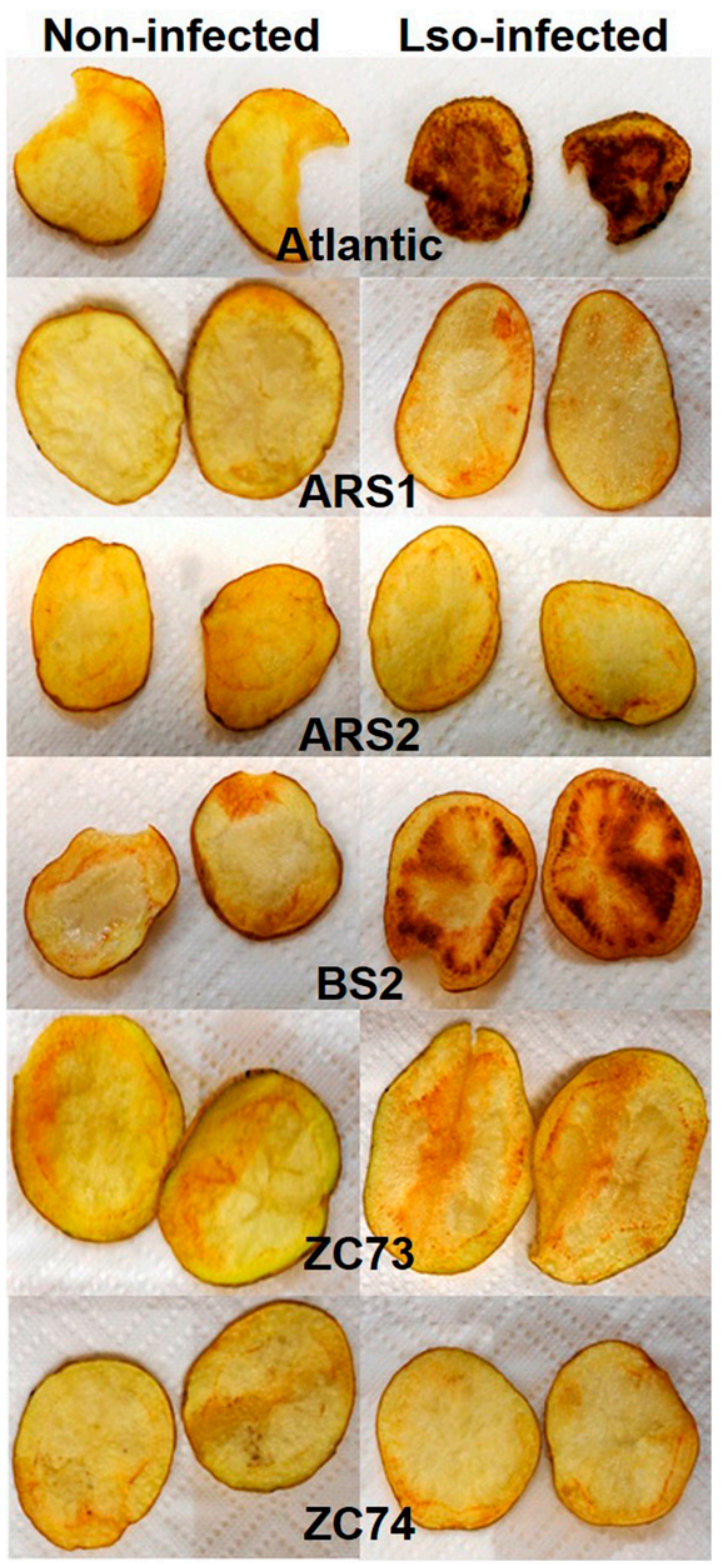

Fig. 1. Examples of fried tuber slices from noninfected and 'Candidatus Liberibacter solanacearum' (Lso)-infected tubers of susceptible (Atlantic) and tolerant entries. compounds using data from both years (with year, year by infection status, year by entry, and the three-way interaction added to model with infection status, entry, and their interaction) as well as each year individually due to significantly effects on compound levels due to year for almost every compound. ANOVAs followed by Tukey's honest significant difference (HSD) tests were performed for each individual compound using only Entry as an independent variable for each other treatment combination (noninfected or infected tubers for each year or both years). This allowed observations to be made regarding differences in chemistry between entries that might affect eventually Lso infection progression, as well as differences that developed when tubers were already infected with Lso.

In order to examine whether fold differences varied between entries, compound levels from five Lso-inoculated tubers were divided by the same compound levels from five noninoculated tubers for either year. The result was fold differences, with values greater than one representing increased levels in Lso-inoculated tubers, and levels less than one representing greater levels in noninoculated tubers. These values were subsequently analyzed by MANOVA with entry as the factor for each year separately for amino acids, sugars, or phenolics. Follow-up ANOVAs on individual compounds were performed, with Tukey's HSD tests utilized to determine pairwise differences between entries. In addition, a mean fold change for all amino acids or phenolics was calculated, and these values were

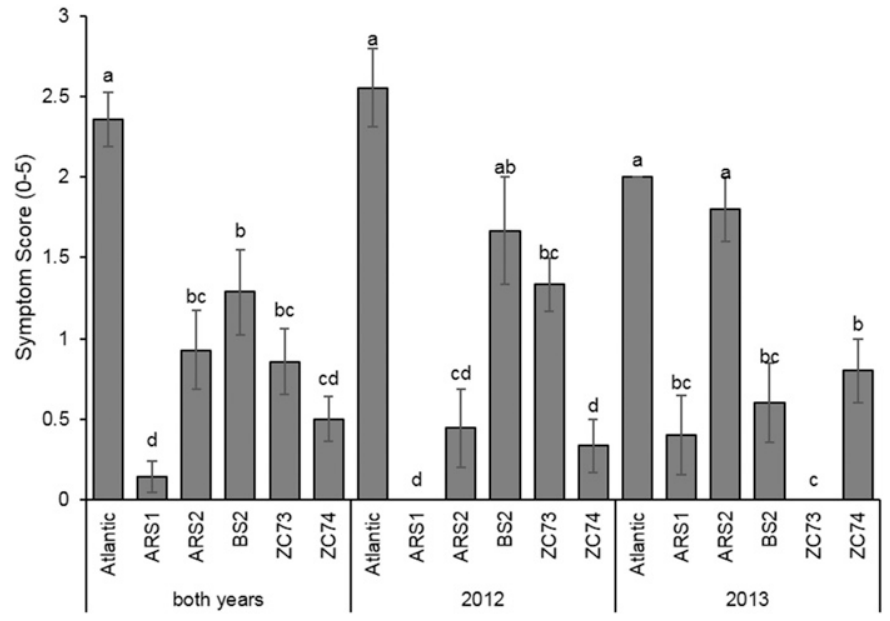

Fig. 2. Mean fried symptom ratings for tubers from each entry for both years, 2012, and 2013. Bars represent standard errors. Different letters represent means that differ significantly by Tukey's honest significant difference test.

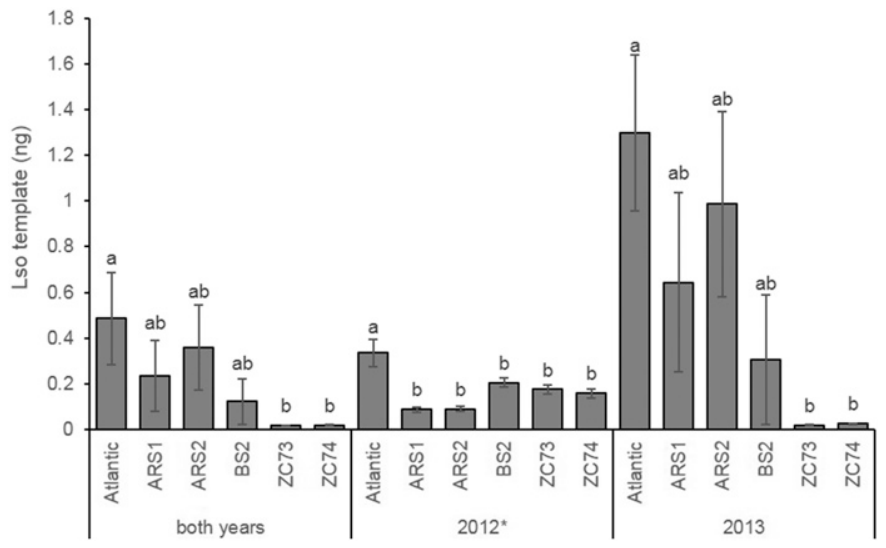

Fig. 3. Mean 'Candidatus Liberibacter solanacearum' titers from each entry for both years, 2012, and 2013. Bars represent standard errors. Different letters represent means that differ significantly by Tukey's honest significant difference test. * indicates that for 2012 , the values represented on the graph are multiplied by 10 to allow for better observation. 
analyzed by ANOVA followed by Tukey's HSD tests with entry as the factor to further compare and visualize fold differences.

Lastly, to analyze relationships between symptoms, Lso titers, and chemicals, Spearman's correlations were performed using only inoculated tubers and data for both years.

\section{RESULTS}

Field screening of potato cultivars for $\mathrm{ZC}$ resistance. During the 4 years of the field screening trial, all the Atlantic control plants in the cages exposed to Lso-infective psyllids produced tubers with very severe fresh and fried ZC symptoms indicating that inoculation during each trial was successful. Furthermore, above ground plant symptoms in Atlantic were observed in all cages and plants generally died soon after ZC symptom development. Plant symptoms included leaf rolling of upper leaves, basal cupping of leaflets, foliar chlorosis, proliferation of axillary buds, shortened internodes, swollen nodes, and leaf scorching. Tuber symptoms from trial entries following Lso infection included collapsed stolons, brown discoloration of the vascular ring, necrotic flecking of internal tissues, and streaking of the medullary ray tissues. A total of 254 out of 283 potato entries had severe ZC tuber symptoms. The remaining 29 entries had ZC symptoms in fresh tubers ranging from light to almost none and this was consistent over multiple years. Lso infection was confirmed in tubers collected from inoculated plants of all 283 lines and the Atlantic controls. Tuber samples of the 29 entries with no symptoms to light symptoms were processed into chips and fried as described by Munyaneza et al. (2007). Of the 29 with no symptoms to light fresh ZC symptoms, five breeding lines (ARS1, ARS2, BS2, ZC73, and ZC74) showed negligible to no $\mathrm{ZC}$ symptoms in fried potato slices. Therefore, tubers for each of the five promising clones, in addition to Atlantic for comparison, were sent to USDA-ARS Parlier in 2012 and 2013 for further symptom assessment and biochemical analyses.

Confirmation of fresh and fried tuber symptoms from susceptible and tolerant entries. Fresh tuber symptoms of putative tolerant clones were significantly less than Atlantic for both years (mean rating of $0.0 \pm 0.0 \mathrm{SE}$ for all tolerant clones except ARS1, which was $0.07 \pm 0.07$, versus $1.43 \pm 0.17$ for Atlantic; $F=$ 48.992; $P<0.001 ; n=84$ for entries; $F=0.150 ; P=0.700 ; n=84$ for year; and $F=0.282 ; P=0.921 ; n=84$ for the entry and year interaction), 2012 (mean rating of $0.0 \pm 0.0 \mathrm{SE}$ for all tolerant clones versus $1.44 \pm 0.24$ for Atlantic; $F=35.579 ; P<0.001 ; n=54)$, and 2013 (mean rating of $0.0 \pm 0.0 \mathrm{SE}$ for all tolerant clones except ARS1, which was $0.20 \pm 0.20$, versus $1.44 \pm 0.24$ for Atlantic; $F=$ $18.880 ; P<0.001 ; n=30)$.

When both years were analyzed together, Atlantic tubers had significantly greater $(F=19.724 ; P<0.001 ; n=84$ for entries; $F=0.849$;

\begin{tabular}{|c|c|c|c|c|c|c|c|c|c|c|c|}
\hline \multirow[b]{2}{*}{ Compound } & \multirow[b]{2}{*}{ Year } & \multirow[b]{2}{*}{ Treatment } & \multirow[b]{2}{*}{ Atlantic } & \multirow[b]{2}{*}{ ARS1 } & \multirow[b]{2}{*}{ ARS2 } & \multirow[b]{2}{*}{ BS2 } & \multirow[b]{2}{*}{$\mathrm{ZC} 73$} & \multirow[b]{2}{*}{ ZC74 } & \multicolumn{3}{|c|}{$F$} \\
\hline & & & & & & & & & $\begin{array}{c}\text { Between } \\
\text { entry }\end{array}$ & $\begin{array}{c}\text { Between } \\
\text { year }\end{array}$ & $\begin{array}{l}\text { Between } \\
\text { treatment }\end{array}$ \\
\hline \multirow[t]{6}{*}{ Alanine } & \multirow[t]{2}{*}{ Both } & Control & $577 \pm 134 \mathrm{c}$ & $525 \pm 100 \mathrm{c}$ & $759 \pm 161 \mathrm{abc}$ & $1110 \pm 160 \mathrm{a}$ & $616 \pm 110 \mathrm{bc}$ & $1040 \pm 190 \mathrm{ab}$ & $4.708 * *$ & $42.580 * * *$ & $12.477 * * *$ \\
\hline & & Lso+ & $756 \pm 71 \mathrm{a}$ & $1160 \pm 230 \mathrm{a}$ & $1050 \pm 260 \mathrm{a}$ & $1420 \pm 210 \mathrm{a}$ & $775 \pm 137 \mathrm{a}$ & $1410 \pm 180 \mathrm{a}$ & $2.844 *$ & 0.071 & \\
\hline & \multirow[t]{2}{*}{2012} & Control & $799 \pm 162 \mathrm{c}$ & $756 \pm 105 c$ & $914 \pm 69 \mathrm{bc}$ & $1460 \pm 180 a b$ & $746 \pm 108 \mathrm{c}$ & $1550 \pm 110 a$ & $8.319^{* * * *}$ & & 0.702 \\
\hline & & Lso+ & $864 \pm 75 b$ & $904 \pm 143 b$ & $872 \pm 104 b$ & $1340 \pm 210 a b$ & $1050 \pm 140 \mathrm{ab}$ & $1610 \pm 180 \mathrm{a}$ & $4.271 * *$ & & \\
\hline & \multirow[t]{2}{*}{2013} & Control & $312 \pm 162$ & $247 \pm 50$ & $574 \pm 345$ & $692 \pm 114$ & $461 \pm 193$ & $430 \pm 100$ & 0.774 & & $9.155^{* *}$ \\
\hline & & Lso+ & $559 \pm 104$ & $1620 \pm 570$ & $1360 \pm 740$ & $1550 \pm 500$ & $274 \pm 86$ & $1040 \pm 350$ & 1.42 & & \\
\hline \multirow[t]{6}{*}{ Asparagine } & Both & Control & $4160 \pm 1440 \mathrm{~b}$ & $10800 \pm 4190 \mathrm{ab}$ & $10600 \pm 4700 \mathrm{ab}$ & $13600 \pm 4900 \mathrm{a}$ & $15200 \pm 5700 \mathrm{a}$ & $13000 \pm 4600 \mathrm{a}$ & $4.661 * *$ & $230.266 * * *$ & $4.285 *$ \\
\hline & & Lso+ & $6600 \pm 2500 \mathrm{ab}$ & $11200 \pm 4300 \mathrm{a}$ & $6630 \pm 3130 \mathrm{ab}$ & $6860 \pm 2650 \mathrm{ab}$ & $8220 \pm 3200 \mathrm{ab}$ & $5420 \pm 2050 \mathrm{~b}$ & $4.685^{* * * *}$ & $370.733 * * *$ & \\
\hline & 2012 & Control & $154 \pm 27 \mathrm{ab}$ & $94 \pm 26 \mathrm{ab}$ & $72 \pm 15 b$ & $194 \pm 51 \mathrm{a}$ & $85 \pm 10 \mathrm{ab}$ & $134 \pm 24 \mathrm{ab}$ & $2.725^{*}$ & & $4.039 *$ \\
\hline & & Lso+ & $356 \pm 56 \mathrm{a}$ & $105 \pm 14 \mathrm{~b}$ & $78 \pm 11 b$ & $190 \pm 33 \mathrm{~b}$ & $93 \pm 12 b$ & $119 \pm 14 b$ & $13.434 * * *$ & & \\
\hline & 2013 & Control & $8970 \pm 860 \mathrm{~b}$ & $23700 \pm 4500 \mathrm{ab}$ & $23200 \pm 6900 \mathrm{ab}$ & $29800 \pm 3600 \mathrm{a}$ & $33200 \pm 5300 \mathrm{a}$ & $28500 \pm 2100 \mathrm{a}$ & $3.804 *$ & & 2.816 \\
\hline & & Lso+ & $17900 \pm 2500$ & $31100 \pm 4100$ & $18400 \pm 5900$ & $18900 \pm 2800$ & $22800 \pm 3100$ & $15000 \pm 1700$ & 2.456 & & \\
\hline Aspartic acid & Both & Control & $5370 \pm 1230$ & $5050 \pm 1340$ & $7990 \pm 2470$ & $6990 \pm 1660$ & $5340 \pm 1340$ & $7220 \pm 1980$ & 1.011 & $80.734 * * *$ & $4.305^{*}$ \\
\hline & & Lso+ & $13000 \pm 1300 \mathrm{a}$ & $5090 \pm 950 \mathrm{~b}$ & $9350 \pm 2370 \mathrm{ab}$ & $9160 \pm 2280 \mathrm{ab}$ & $7060 \pm 2140 \mathrm{ab}$ & $8890 \pm 1850 \mathrm{ab}$ & $3.194 *$ & $28.919 * * *$ & \\
\hline & 2012 & Control & $8520 \pm 1060$ & $8250 \pm 1450$ & $12600 \pm 3600$ & $11400 \pm 1300$ & $8350 \pm 1600$ & $11800 \pm 2300$ & 0.926 & & 0.753 \\
\hline & & Lso+ & $12600 \pm 1940$ & $6580 \pm 1200$ & $13100 \pm 3000$ & $13200 \pm 2700$ & $10000 \pm 2900$ & $12600 \pm 1900$ & 1.2 & & \\
\hline & 2013 & Control & $1580 \pm 260$ & $1210 \pm 80$ & $2450 \pm 430$ & $1750 \pm 250$ & $1730 \pm 210$ & $1740 \pm 180$ & 2.41 & & $66.133^{* * * *}$ \\
\hline & & Lso+ & $13700 \pm 1300 \mathrm{a}$ & $2400 \pm 400 \mathrm{~b}$ & $2640 \pm 640 \mathrm{~b}$ & $1940 \pm 310 \mathrm{~b}$ & $1820 \pm 350 \mathrm{~b}$ & $2170 \pm 360 \mathrm{~b}$ & $50.125 * * *$ & & \\
\hline Glutamic acid & Both & Control & $2340 \pm 310$ & $1770 \pm 420$ & $2100 \pm 570$ & $1800 \pm 210$ & $2140 \pm 390$ & $1750 \pm 260$ & 0.453 & 3.51 & 0.502 \\
\hline & & Lso+ & $4900 \pm 1210$ & $1390 \pm 330$ & $2160 \pm 590$ & $2680 \pm 730$ & $1960 \pm 700$ & $2410 \pm 570$ & 2.182 & $26.183^{* * *}$ & \\
\hline & 2012 & Control & $2330 \pm 240$ & $2370 \pm 700$ & $2450 \pm 1030$ & $2130 \pm 310$ & $2320 \pm 630$ & $1920 \pm 470$ & 0.098 & & $6.241 *$ \\
\hline & & Lso+ & $7060 \pm 1440 \mathrm{a}$ & $1770 \pm 470 \mathrm{~b}$ & $3080 \pm 750 a b$ & $3680 \pm 990 a b$ & $2520 \pm 1060 b$ & $3280 \pm 730 a b$ & $3.681 *$ & & \\
\hline & 2013 & Control & $2360 \pm 670$ & $1060 \pm 130$ & $1680 \pm 350$ & $1410 \pm 210$ & $1930 \pm 460$ & $1540 \pm 170$ & 1.378 & & $23.365^{* * * *}$ \\
\hline & & Lso+ & $1030 \pm 250$ & $717 \pm 142$ & $513 \pm 155$ & $869 \pm 118$ & $946 \pm 163$ & $838 \pm 272$ & 0.894 & & \\
\hline Glutamine & Both & Control & $2980 \pm 1270$ & $2780 \pm 800$ & $5660 \pm 3310$ & $4490 \pm 1420$ & $5150 \pm 2000$ & $4270 \pm 1290$ & 0.76 & $40.461 * * *$ & $16.553^{* * *}$ \\
\hline & & Lso+ & $8550 \pm 2980$ & $8610 \pm 4140$ & $7520 \pm 4040$ & $8570 \pm 3600$ & $3840 \pm 1490$ & $4170 \pm 1580$ & 1.881 & $84.066 * * *$ & \\
\hline & 2012 & Control & $746 \pm 14$ & $880 \pm 134$ & $740 \pm 69$ & $868 \pm 52$ & $640 \pm 22$ & $748 \pm 26$ & 1.815 & & 0.063 \\
\hline & & Lso+ & $870 \pm 109 a b$ & $838 \pm 84 a b$ & $1000 \pm 120 \mathrm{a}$ & $762 \pm 89 \mathrm{ab}$ & $608 \pm 60 \mathrm{~b}$ & $617 \pm 73 \mathrm{~b}$ & $2.785 *$ & & \\
\hline & 2013 & Control & $5660 \pm 2360$ & $5050 \pm 1070$ & $11600 \pm 6700$ & $8850 \pm 1560$ & $10600 \pm 2900$ & $8500 \pm 960$ & 0.643 & & $10.664 * *$ \\
\hline & & Lso+ & $22400 \pm 2600$ & $22600 \pm 8950$ & $19200 \pm 9800$ & $22600 \pm 6600$ & $9650 \pm 2600$ & $10600 \pm 2600$ & 0.94 & & \\
\hline Glycine & Both & Control & $149 \pm 35 b$ & $139 \pm 26 b$ & $139 \pm 20 \mathrm{~b}$ & $229 \pm 32 \mathrm{a}$ & $137 \pm 21 \mathrm{~b}$ & $221 \pm 47 \mathrm{a}$ & $8.874 * * *$ & $231.410^{* * * *}$ & $22.579 * * *$ \\
\hline & & Lso+ & $391 \pm 31 \mathrm{a}$ & $180 \pm 20 \mathrm{c}$ & $148 \pm 12 c$ & $262 \pm 36 b$ & $178 \pm 27 \mathrm{c}$ & $219 \pm 41 b c$ & $22.432 * * *$ & $59.090 * * *$ & \\
\hline & 2012 & Control & $238 \pm 32 b c$ & $203 \pm 24 c$ & $189 \pm 17 \mathrm{c}$ & $313 \pm 21 a b$ & $191 \pm 16 \mathrm{c}$ & $352 \pm 24 \mathrm{a}$ & $9.058^{* * *}$ & & 3.376 \\
\hline & & Lso+ & $371 \pm 36 \mathrm{a}$ & $203 \pm 27 \mathrm{c}$ & $169 \pm 11 \mathrm{c}$ & $345 \pm 30 \mathrm{ab}$ & $246 \pm 14 b c$ & $318 \pm 28 \mathrm{bc}$ & $9.880 * * *$ & & \\
\hline & 2013 & Control & $42.2 \pm 4.1 \mathrm{~b}$ & $63.0 \pm 9.8 \mathrm{~b}$ & $78.8 \pm 13.5 \mathrm{ab}$ & $129 \pm 23 \mathrm{a}$ & $73.2 \pm 6.5 \mathrm{~b}$ & $63.0 \pm 10.1 \mathrm{~b}$ & 5.509 & & $34.004 * * *$ \\
\hline & & Lso+ & $426 \pm 62 \mathrm{a}$ & $139 \pm 16 \mathrm{bc}$ & $112 \pm 16 b c$ & $111 \pm 7 \mathrm{~b}$ & $56.6 \pm 14.5 \mathrm{bc}$ & $40.6 \pm 1.7 \mathrm{c}$ & $25.841 * * *$ & & \\
\hline Histidine & Both & Control & $168 \pm 46$ & $255 \pm 92$ & $365 \pm 179$ & $284 \pm 35$ & $346 \pm 109$ & $421 \pm 207$ & 0.438 & 1.319 & $12.403 * * *$ \\
\hline & & Lso+ & $1550 \pm 330 \mathrm{a}$ & $364 \pm 125 b$ & $671 \pm 209 a b$ & $841 \pm 273 \mathrm{ab}$ & $332 \pm 121 b$ & $721 \pm 201 \mathrm{ab}$ & $3.159 *$ & 1.219 & \\
\hline & 2012 & Control & $204 \pm 68$ & $338 \pm 164$ & $406 \pm 294$ & $293 \pm 49$ & $319 \pm 129$ & $615 \pm 374$ & 0.426 & & $6.443 *$ \\
\hline & & Lso+ & $1680 \pm 510$ & $252 \pm 135$ & $754 \pm 287$ & $939 \pm 411$ & $386 \pm 185$ & $917 \pm 291$ & 2.365 & & \\
\hline & 2013 & Control & $125 \pm 61$ & $155 \pm 33$ & $315 \pm 214$ & $274 \pm 54$ & $378 \pm 199$ & $188 \pm 34$ & 0.623 & & $25.841 * * *$ \\
\hline & & Lso+ & $1300 \pm 90 \mathrm{a}$ & $565 \pm 246 \mathrm{ab}$ & $522 \pm 304 \mathrm{ab}$ & $663 \pm 237 \mathrm{ab}$ & $234 \pm 80 \mathrm{~b}$ & $368 \pm 122 b$ & $3.478^{*}$ & & \\
\hline Isoleucine & Both & Control & $325 \pm 86 \mathrm{~b}$ & $165 \pm 19 \mathrm{~b}$ & $159 \pm 20 \mathrm{~b}$ & $724 \pm 106 \mathrm{a}$ & $304 \pm 40 \mathrm{~b}$ & $342 \pm 45 \mathrm{~b}$ & $12.712^{* * *}$ & 3.402 & $34.105 * * *$ \\
\hline & & Lso+ & $997 \pm 102 \mathrm{a}$ & $468 \pm 90 b$ & $334 \pm 52 \mathrm{~b}$ & $768 \pm 114 a$ & $243 \pm 37 b$ & $273 \pm 28 b$ & $30.684 * * *$ & $5.377 *$ & \\
\hline & 2012 & Control & $463 \pm 136 b$ & $175 \pm 25 b$ & $120 \pm 20 b$ & $883 \pm 161 \mathrm{a}$ & $236 \pm 21 b$ & $372 \pm 57 \mathrm{~b}$ & $9.553 * * *$ & & $4.097 *$ \\
\hline & & Lso+ & $779 \pm 80 \mathrm{a}$ & $258 \pm 31 \mathrm{~b}$ & $239 \pm 49 \mathrm{~b}$ & $926 \pm 153 \mathrm{a}$ & $312 \pm 42 b$ & $329 \pm 31 \mathrm{~b}$ & $14.973^{* * *}$ & & \\
\hline & 2013 & Control & $159 \pm 13 \mathrm{~b}$ & $153 \pm 31 \mathrm{~b}$ & $206 \pm 24 b$ & $534 \pm 83 a$ & $386 \pm 70 \mathrm{ab}$ & $305 \pm 74 \mathrm{ab}$ & $7.102 * * *$ & & $59.662 * * *$ \\
\hline & & Lso+ & $1390 \pm 110 \mathrm{a}$ & $848 \pm 120 \mathrm{~b}$ & $506 \pm 69 c$ & $483 \pm 43 \mathrm{~cd}$ & $119 \pm 19 \mathrm{e}$ & $173 \pm 5 \mathrm{de}$ & $39.620^{* * *}$ & & \\
\hline Leucine & Both & Control & $278 \pm 70 \mathrm{bc}$ & $172 \pm 32 \mathrm{c}$ & $164 \pm 13 \mathrm{c}$ & $453 \pm 85 a$ & $194 \pm 22 \mathrm{c}$ & $366 \pm 66 \mathrm{ab}$ & $9.156 * * *$ & $60.135^{* * *}$ & $26.920 * * *$ \\
\hline & & Lso+ & $673 \pm 47 \mathrm{a}$ & $333 \pm 42 c$ & $270 \pm 20 \mathrm{c}$ & $548 \pm 86 \mathrm{ab}$ & $250 \pm 37 \mathrm{c}$ & $393 \pm 70 \mathrm{bc}$ & $17.774 * * *$ & $56.546^{* * * *}$ & \\
\hline & 2012 & Control & $413 \pm 100 \mathrm{ab}$ & $252 \pm 30 \mathrm{~b}$ & $177 \pm 18 b$ & $639 \pm 102 \mathrm{a}$ & $249 \pm 17 b$ & $535 \pm 51 \mathrm{a}$ & $8.192 * * *$ & & $12.289 * * *$ \\
\hline & & Lso+ & $692 \pm 59 \mathrm{a}$ & $400 \pm 52 b c$ & $265 \pm 25 \mathrm{c}$ & $733 \pm 82 \mathrm{a}$ & $337 \pm 30 \mathrm{bc}$ & $557 \pm 53 \mathrm{ab}$ & $12.982 * * *$ & & \\
\hline & 2013 & Control & $115 \pm 14 \mathrm{~b}$ & $77.4 \pm 9.7 \mathrm{~b}$ & $149 \pm 18 \mathrm{ab}$ & $231 \pm 34 \mathrm{a}$ & $129 \pm 13 \mathrm{ab}$ & $164 \pm 36 \mathrm{ab}$ & $4.923 * *$ & & $35.239 * * *$ \\
\hline & & Lso+ & $639 \pm 83 a$ & $213 \pm 26 b c$ & $279 \pm 38 \mathrm{~b}$ & $216 \pm 20 b c$ & $94.6 \pm 14.2 \mathrm{c}$ & $96.6 \pm 1.1 \mathrm{c}$ & $25.431 * * *$ & & \\
\hline
\end{tabular}


$P=0.360 ; n=84$ for year; and $F=10.130 ; P<0.001 ; n=84$ for the entry and year interaction) ZC fried symptoms than all other entries, and BS2 tubers had greater fried symptoms than ARS1 and ZC74 (Fig. 2A). In 2012, Atlantic tubers exhibited greater fried symptoms $(F=$ 19.904; $P<0.001 ; n=54$ ) when infected with Lso than all of the putative tolerant breeding clones except BS2, with ARS1 and ZC74 exhibiting the least symptoms (Figs. 1 and 2). In 2013, Atlantic tubers exhibited greater fried symptoms $(F=19.040 ; P<0.001 ; n=30)$ when infected with Lso than all of the putative tolerant clones except ARS2, with ZC73 exhibiting the least symptoms (Fig. 2).

All noninoculated tubers used in this study had $\mathrm{Ct}$ values from the qPCR analyses equivalent to negative controls and thus were deemed noninfected. Of Lso-inoculated plants, tubers from the Atlantic cultivar had greater Lso titers than ZC73 and ZC74 when both years were analyzed together $(F=6.265 ; P<0.001 ; n=84$ for entry; $F=37.720 ; P<0.001 ; n=84$ for year; and $F=6.128 ; P<$ $0.001 ; n=84$ for the entry and year interaction), and tubers from 2013 had greater titers than those from 2012 (Fig. 3). For 2012, Atlantic tubers had greater Lso titers than all other entries $(F=$ 9.367; $P<0.001 ; n=54$ ) (Fig. 3). However, even though ANOVA suggested a significant difference in Lso titers due to entry in 2013 $(F=3.213 ; P=0.023 ; n=30)$, follow-up Tukey's HSD tests failed to separate means between entries, albeit Atlantic had marginally $(P<$ 0.06) increased titers over ZC73 and ZC74 (Fig. 3).

Fresh and fried symptoms were positively correlated with each other $(\rho=0.523 ; P<0.001 ; n=84)$. Likewise, Lso titers were positively correlated with both fresh $(\rho=0.319 ; P=0.003 ; n=84)$ and fried symptoms $(\rho=0.494 ; P<0.001 ; n=84)$.

Amino acid responses in susceptible and tolerant entries. A total of 18 amino acids (alanine, asparagine, aspartic acid, glutamic acid, glutamine, glycine, histidine, isoleucine, leucine, lysine, methionine, phenylalanine, proline, serine, threonine, tryptophan, tyrosine, and valine) were analyzed in tubers in both 2012 and 2013.

Entry, infection status, and year significantly affected overall levels of amino acids (Pillai's trace $\Delta=3.019 ; F=8.986 ; P<0.001$; $n=150$ for entry; $\Delta=0.827 ; F=27.144 ; P<0.001 ; n=150$ for infection status; and $\Delta=0.941 ; F=90.657 ; P<0.001 ; n=150$ for year) and all interactions between variables were significant (Pillai's trace $\Delta=2.099 ; F=4.267 ; P<0.001 ; n=150$ for entry and infection status; $\Delta=2.787 ; F=7.423 ; P<0.001 ; n=150$ for entry and year; $\Delta=0.852 ; F=32.834 ; P<0.001 ; n=150$ for infection status and year; and $\Delta=1.923 ; F=3.684 ; P<0.001 ; n=$ 150 for entry, infection status and year).

Entry and infection status significantly affected overall levels of amino acids in both 2012 (Pillai's trace $\Delta=3.200 ; F=6.420 ; P<$ $0.001 ; n=90$ for entry; $\Delta=0.486 ; F=3.200 ; P<0.001 ; n=90$ for infection status $)$ and $2013(\Delta=4.196 ; F=10.143 ; P<0.001 ; n=60$ for entry; $\Delta=0.984 ; F=104.480 ; P<0.001 ; n=60$ for infection status). However, in both 2012 (Pillai's trace $\Delta=1.541 ; F=1.609$; $P=0.001 ; n=90)$ and $2013(\Delta=3.640 ; F=5.206 ; P<0.001 ; n=60)$, MANOVAs suggested significant differences due to the entry and infection interaction.

TABLE 1. (continued from preceding page)

\begin{tabular}{|c|c|c|c|c|c|c|c|c|c|c|c|}
\hline \multirow[b]{2}{*}{ Compound } & \multirow[b]{2}{*}{ Year } & \multirow[b]{2}{*}{ Treatment } & \multirow[b]{2}{*}{ Atlantic } & \multirow[b]{2}{*}{ ARS1 } & \multirow[b]{2}{*}{ ARS2 } & \multirow[b]{2}{*}{ BS2 } & \multirow[b]{2}{*}{ ZC73 } & \multirow[b]{2}{*}{ ZC74 } & \multicolumn{3}{|c|}{$F$} \\
\hline & & & & & & & & & $\begin{array}{c}\text { Between } \\
\text { entry }\end{array}$ & $\begin{array}{l}\text { Between } \\
\text { year }\end{array}$ & $\begin{array}{l}\text { Between } \\
\text { treatment }\end{array}$ \\
\hline \multirow[t]{6}{*}{ Lysine } & \multirow[t]{2}{*}{ Both } & Control & $308 \pm 75$ & $395 \pm 125$ & $612 \pm 271$ & $660 \pm 58$ & $613 \pm 183$ & $745 \pm 317$ & 0.65 & 1.302 & $18.732 * * *$ \\
\hline & & Lso+ & $3440 \pm 720 \mathrm{a}$ & $890 \pm 294 b$ & $1090 \pm 337 b$ & $1880 \pm 560 \mathrm{ab}$ & $539 \pm 208 b$ & $1650 \pm 430 a b$ & $4.045^{* *}$ & 0.24 & \\
\hline & \multirow[t]{2}{*}{2012} & Control & $306 \pm 57$ & $518 \pm 222$ & $643 \pm 434$ & $633 \pm 59$ & $740 \pm 300$ & $1010 \pm 580$ & 0.489 & & $8.113 * *$ \\
\hline & & Lso+ & $3740 \pm 1130 a$ & $486 \pm 268 b$ & $949 \pm 355 \mathrm{ab}$ & $2070 \pm 840 \mathrm{ab}$ & $687 \pm 316 b$ & $1990 \pm 622 a b$ & $3.324 * *$ & & \\
\hline & \multirow[t]{2}{*}{2013} & Control & $312 \pm 162$ & $247 \pm 50$ & $574 \pm 345$ & $692 \pm 114$ & $461 \pm 193$ & $430 \pm 100$ & 0.774 & & $24.417 * * *$ \\
\hline & & Lso+ & $2920 \pm 157 \mathrm{a}$ & $1620 \pm 570 a b$ & $1360 \pm 740 a b$ & $1550 \pm 500 \mathrm{ab}$ & $274 \pm 86 b$ & $1040 \pm 350 a b$ & $3.501 *$ & & \\
\hline \multirow[t]{6}{*}{ Methionine } & Both & Control & $104 \pm 12$ & $145 \pm 30$ & $213 \pm 70$ & $194 \pm 54$ & $202 \pm 36$ & $181 \pm 37$ & 1.299 & $9.887 * *$ & $33.010^{* * *}$ \\
\hline & & Lso+ & $615 \pm 141 \mathrm{a}$ & $198 \pm 59 \mathrm{~b}$ & $193 \pm 49 b$ & $238 \pm 50 \mathrm{~b}$ & $196 \pm 52 \mathrm{~b}$ & $208 \pm 32 b$ & $25.106 * * *$ & $72.873 * * *$ & \\
\hline & 2012 & Control & $90.5 \pm 15.5$ & $140 \pm 48$ & $121 \pm 64$ & $57.7 \pm 7.3$ & $180 \pm 45$ & $177 \pm 67$ & 1.047 & & $24.417 * * *$ \\
\hline & & Lso+ & $256 \pm 65$ & $64.3 \pm 23.1$ & $119 \pm 44$ & $148 \pm 50$ & $196 \pm 81$ & $216 \pm 49$ & 1.612 & & \\
\hline & 2013 & Control & $120 \pm 16$ & $151 \pm 37$ & $324 \pm 124$ & $357 \pm 59$ & $229 \pm 61$ & $187 \pm 32$ & 2.156 & & $43.899 * * *$ \\
\hline & & Lso+ & $1260 \pm 47 \mathrm{a}$ & $439 \pm 86 b$ & $327 \pm 88 \mathrm{~b}$ & $400 \pm 59 \mathrm{~b}$ & $196 \pm 34 b$ & $194 \pm 24 b$ & $42.866^{* * *}$ & & \\
\hline Phenylalanine & Both & Control & $225 \pm 49 \mathrm{a}$ & $150 \pm 31 \mathrm{c}$ & $160 \pm 34 c$ & $468 \pm 52 b$ & $302 \pm 39 c$ & $315 \pm 82 \mathrm{c}$ & $24.640^{* * *}$ & $26.192 * * *$ & $68.789^{* * *}$ \\
\hline & & Lso+ & $1390 \pm 240 \mathrm{a}$ & $279 \pm 52 c$ & $418 \pm 105 \mathrm{bc}$ & $721 \pm 123 b$ & $238 \pm 52 \mathrm{c}$ & $365 \pm 54 \mathrm{c}$ & $35.309 * * *$ & $41.739 * * *$ & \\
\hline & 2012 & Control & $277 \pm 83 \mathrm{a}$ & $116 \pm 36 a$ & $114 \pm 51 \mathrm{a}$ & $458 \pm 77 \mathrm{a}$ & $305 \pm 60 \mathrm{a}$ & $378 \pm 139 a$ & $2.904 *$ & & $4.547 *$ \\
\hline & & Lso+ & $812 \pm 159 a$ & $187 \pm 51 \mathrm{~b}$ & $217 \pm 41 b$ & $587 \pm 165 \mathrm{ab}$ & $268 \pm 79 b$ & $351 \pm 78 \mathrm{~b}$ & $5.272 * * *$ & & \\
\hline & 2013 & Control & $164 \pm 31 \mathrm{~b}$ & $191 \pm 52 \mathrm{~b}$ & $215 \pm 33 \mathrm{~b}$ & $480 \pm 77 \mathrm{a}$ & $297 \pm 54 \mathrm{ab}$ & $240 \pm 72 a b$ & $4.195^{* *}$ & & $106.885^{* * * *}$ \\
\hline & & Lso+ & $2420 \pm 170 \mathrm{a}$ & $445 \pm 70 \mathrm{bcd}$ & $779 \pm 210 b c$ & $961 \pm 134 \mathrm{~b}$ & $185 \pm 38 \mathrm{~d}$ & $389 \pm 72 \mathrm{~cd}$ & $38.821 * * *$ & & \\
\hline Proline & Both & Control & $636 \pm 228 \mathrm{c}$ & $2110 \pm 640 a b$ & $1020 \pm 280 \mathrm{bc}$ & $3380 \pm 900 \mathrm{a}$ & $718 \pm 135 b c$ & $1740 \pm 450 \mathrm{bc}$ & $7.738^{* * *}$ & $44.371 * * *$ & $5.735^{*}$ \\
\hline & & Lso+ & $4360 \pm 430 \mathrm{a}$ & $1650 \pm 310 b c$ & $1270 \pm 270 \mathrm{bc}$ & $2510 \pm 560 \mathrm{~b}$ & $1150 \pm 250 \mathrm{c}$ & $1250 \pm 170 \mathrm{bc}$ & $15.558 * * *$ & 1.046 & \\
\hline & 2012 & Control & $925 \pm 391 \mathrm{c}$ & $3630 \pm 690 a b$ & $1110 \pm 500 \mathrm{bc}$ & $5450 \pm 1030 \mathrm{a}$ & $943 \pm 203 c$ & $2720 \pm 570 \mathrm{bc}$ & $8.797 * * *$ & & 1.099 \\
\hline & & Lso+ & $3860 \pm 580 \mathrm{a}$ & $1700 \pm 490 \mathrm{bc}$ & $849 \pm 262 c$ & $3260 \pm 770 \mathrm{ab}$ & $1620 \pm 270 \mathrm{bc}$ & $1520 \pm 210 \mathrm{bc}$ & $5.939 * * *$ & & \\
\hline & 2013 & Control & $291 \pm 33 \mathrm{a}$ & $282 \pm 69 \mathrm{a}$ & $910 \pm 203 a$ & $901 \pm 267 \mathrm{a}$ & $448 \pm 71 \mathrm{a}$ & $562 \pm 137 \mathrm{a}$ & $3.382 *$ & & $107.638 * * *$ \\
\hline & & Lso+ & $5280 \pm 422 \mathrm{a}$ & $1570 \pm 130 \mathrm{bc}$ & $2030 \pm 450 \mathrm{~b}$ & $1170 \pm 70 \mathrm{bcd}$ & $297 \pm 36 \mathrm{~d}$ & $769 \pm 34 \mathrm{~cd}$ & $46.549 * * *$ & & \\
\hline Serine & Both & Control & $1650 \pm 200 \mathrm{c}$ & $3000 \pm 530 \mathrm{ab}$ & $3270 \pm 490 \mathrm{ab}$ & $3590 \pm 480 \mathrm{a}$ & $2630 \pm 360 a b c$ & $2410 \pm 300 b c$ & $6.890 * * *$ & $49.665^{* * * *}$ & $24.682 * * *$ \\
\hline & & Lso+ & $5310 \pm 790 \mathrm{a}$ & $2990 \pm 460 \mathrm{bc}$ & $2860 \pm 360 \mathrm{c}$ & $3940 \pm 400 \mathrm{~b}$ & $2020 \pm 180 \mathrm{c}$ & $2760 \pm 200 \mathrm{c}$ & $30.940 * * *$ & $94.464 * * *$ & \\
\hline & 2012 & Control & $1900 \pm 320$ & $1610 \pm 270$ & $2370 \pm 320$ & $2480 \pm 150$ & $1780 \pm 170$ & $1980 \pm 150$ & 1.974 & & $10.932 * *$ \\
\hline & & Lso+ & $3280 \pm 320 \mathrm{a}$ & $1870 \pm 210 \mathrm{~b}$ & $2350 \pm 280 \mathrm{ab}$ & $3190 \pm 380 \mathrm{a}$ & $2150 \pm 270 \mathrm{ab}$ & $2640 \pm 270 a b$ & $3.672 * *$ & & \\
\hline & 2013 & Control & $1360 \pm 130 \mathrm{~b}$ & $4670 \pm 410 \mathrm{a}$ & $4350 \pm 800 \mathrm{a}$ & $4910 \pm 650 \mathrm{a}$ & $3650 \pm 460 a b$ & $2920 \pm 600 \mathrm{ab}$ & $5.904 * *$ & & $11.707 * *$ \\
\hline & & Lso+ & $8960 \pm 380 \mathrm{a}$ & $5010 \pm 440 \mathrm{~b}$ & $3790 \pm 750 \mathrm{bc}$ & $5290 \pm 480 \mathrm{~b}$ & $1780 \pm 110 \mathrm{~d}$ & $2970 \pm 290 \mathrm{~cd}$ & $30.553 * * *$ & & \\
\hline Threonine & Both & Control & $210 \pm 33 \mathrm{~b}$ & $432 \pm 57 \mathrm{ab}$ & $514 \pm 102 \mathrm{a}$ & $502 \pm 48 \mathrm{a}$ & $323 \pm 32 \mathrm{ab}$ & $281 \pm 39 a b$ & $5.093 * * *$ & 0.075 & 28.129 *** \\
\hline & & Lso+ & $950 \pm 104 \mathrm{a}$ & $610 \pm 113 b$ & $607 \pm 80 \mathrm{~b}$ & $614 \pm 49 \mathrm{~b}$ & $286 \pm 52 c$ & $311 \pm 43 \mathrm{c}$ & $16.788 * * *$ & 2.993 & \\
\hline & 2012 & Control & $282 \pm 40$ & $360 \pm 42$ & $496 \pm 128$ & $451 \pm 32$ & $296 \pm 35$ & $340 \pm 54$ & 1.776 & & $11.535 * *$ \\
\hline & & Lso+ & $820 \pm 122 \mathrm{a}$ & $363 \pm 58 \mathrm{~b}$ & $610 \pm 102 \mathrm{ab}$ & $605 \pm 72 \mathrm{ab}$ & $386 \pm 57 \mathrm{~b}$ & $385 \pm 52 b$ & $5.011 * * *$ & & \\
\hline & 2013 & Control & $124 \pm 13 \mathrm{~b}$ & $517 \pm 110 a b$ & $534 \pm 180 \mathrm{ab}$ & $562 \pm 96 \mathrm{a}$ & $355 \pm 58 \mathrm{ab}$ & $209 \pm 38 \mathrm{ab}$ & $3.487 *$ & & $15.585^{* * * *}$ \\
\hline & & Lso+ & $1180 \pm 150 \mathrm{a}$ & $1050 \pm 170 a b$ & $600 \pm 144 b c$ & $629 \pm 55 \mathrm{bc}$ & $106 \pm 14 \mathrm{~d}$ & $179 \pm 17 \mathrm{~cd}$ & $15.295 * * *$ & & \\
\hline Tryptophan & Both & Control & $76.5 \pm 17.2$ & $64.4 \pm 15.2$ & $140 \pm 64$ & $165 \pm 36$ & $95.0 \pm 38.8$ & $84.5 \pm 16.2$ & 1.771 & $14.148 * * *$ & $33.798 * * *$ \\
\hline & & Lso+ & $333 \pm 95 \mathrm{a}$ & $282 \pm 88 \mathrm{ab}$ & $163 \pm 69 a b c$ & $243 \pm 80 a b c$ & $63.1 \pm 8.2 \mathrm{c}$ & $134 \pm 35 \mathrm{bc}$ & $7.553 * * *$ & $78.518^{* * *}$ & \\
\hline & 2012 & Control & $74.3 \pm 7.5 \mathrm{a}$ & $47.2 \pm 5.6 \mathrm{a}$ & $63.8 \pm 14.8 \mathrm{a}$ & $76.5 \pm 15.0 \mathrm{a}$ & $32.8 \pm 8.0 \mathrm{a}$ & $62.0 \pm 7.7 \mathrm{a}$ & $2.554 *$ & & 1.123 \\
\hline & & Lso+ & $88.3 \pm 12.3$ & $149 \pm 84$ & $48.2 \pm 15.9$ & $73.8 \pm 6.0$ & $61.2 \pm 9.9$ & $53.4 \pm 9.9$ & 1.08 & & \\
\hline & 2013 & Control & $79.0 \pm 39.3$ & $85.0 \pm 32.0$ & $230 \pm 135$ & $271 \pm 40$ & $170 \pm 75$ & $112 \pm 32$ & 1.308 & & $23.972 * * *$ \\
\hline & & Lso+ & $774 \pm 73 \mathrm{a}$ & $520 \pm 154 \mathrm{ab}$ & $370 \pm 160 \mathrm{ab}$ & $547 \pm 147 \mathrm{ab}$ & $66.4 \pm 16.0 \mathrm{~b}$ & $278 \pm 49 a b$ & $4.541 * *$ & & \\
\hline Tyrosine & Both & Control & $48.5 \pm 14.4$ & $53.0 \pm 23.8$ & $35.9 \pm 12.0$ & $46.8 \pm 12.2$ & $56.9 \pm 20.0$ & $69.1 \pm 30.3$ & 0.318 & $17.268^{* * * *}$ & $7.032 * *$ \\
\hline & & Lso+ & $231 \pm 60 a$ & $42.9 \pm 12.0 \mathrm{~b}$ & $57.5 \pm 14.1 \mathrm{~b}$ & $113 \pm 37 \mathrm{ab}$ & $82.7 \pm 36.1 \mathrm{~b}$ & $157 \pm 50 \mathrm{ab}$ & $2.525 *$ & $21.367 * * *$ & \\
\hline & 2012 & Control & $57.7 \pm 22.5$ & $91.5 \pm 37.7$ & $53.5 \pm 19.4$ & $74.7 \pm 13.3$ & $80.0 \pm 34.3$ & $118 \pm 48$ & 0.571 & & $8.611 * *$ \\
\hline & & Lso+ & $336 \pm 72 \mathrm{a}$ & $56.2 \pm 17.3 \mathrm{~b}$ & $77.0 \pm 18.3 \mathrm{~b}$ & $160 \pm 52 \mathrm{ab}$ & $112 \pm 55 \mathrm{~b}$ & $229 \pm 67 \mathrm{ab}$ & $4.192 * *$ & & \\
\hline & 2013 & Control & $37.6 \pm 18.1$ & $6.80 \pm 1.56$ & $14.8 \pm 5.3$ & $13.4 \pm 6.0$ & $29.2 \pm 9.0$ & $10.0 \pm 4.2$ & 1.778 & & $4.215 *$ \\
\hline & & Lso+ & $40.6 \pm 6.4$ & $19.0 \pm 3.9$ & $22.4 \pm 11.0$ & $29.8 \pm 7.0$ & $30.2 \pm 5.1$ & $27.8 \pm 7.4$ & 1.09 & & \\
\hline Valine & Both & Control & $743 \pm 186 b$ & $465 \pm 64 \mathrm{~b}$ & $380 \pm 33 b$ & $2150 \pm 310 a$ & $633 \pm 64 b$ & $864 \pm 121 b$ & $22.262 * * *$ & $16.232 * * *$ & $22.712 * * *$ \\
\hline & & Lso+ & $1720 \pm 143 b$ & $1280 \pm 270 \mathrm{bc}$ & $759 \pm 90 \mathrm{c}$ & $2400 \pm 340 \mathrm{a}$ & $806 \pm 145 c$ & $769 \pm 80 \mathrm{c}$ & $15.442 * * *$ & 1.438 & \\
\hline & 2012 & Control & $1120 \pm 250 \mathrm{~b}$ & $558 \pm 86 \mathrm{~b}$ & $352 \pm 28 \mathrm{~b}$ & $2720 \pm 429 a$ & $619 \pm 48 \mathrm{~b}$ & $1070 \pm 134 \mathrm{~b}$ & $16.079 * * *$ & & $4.362 *$ \\
\hline & & Lso+ & $1920 \pm 190 \mathrm{~b}$ & $619 \pm 68 c$ & $602 \pm 86 c$ & $2900 \pm 450 \mathrm{a}$ & $1100 \pm 150 b c$ & $925 \pm 86 \mathrm{c}$ & $17.316^{* * *}$ & & \\
\hline & 2013 & Control & $294 \pm 15 \mathrm{~b}$ & $355 \pm 78 \mathrm{~b}$ & $413 \pm 67 b$ & $1470 \pm 230 \mathrm{a}$ & $650 \pm 137 \mathrm{~b}$ & $621 \pm 164 \mathrm{~b}$ & $10.160 * * *$ & & $43.469 * * *$ \\
\hline & & Lso+ & $1360 \pm 110 \mathrm{~b}$ & $2480 \pm 310 \mathrm{a}$ & $1040 \pm 130 \mathrm{bc}$ & $1500 \pm 140 \mathrm{~b}$ & $269 \pm 20 \mathrm{~d}$ & $489 \pm 21 \mathrm{~cd}$ & $25.809 * * *$ & & \\
\hline
\end{tabular}


Due to all interactions being significant, subsequent ANOVAs were conducted with treatment combinations separated out. ANOVAs suggested that infection status significantly affected most amino acids with the exception of alanine, aspartic acid, glutamine, glycine, proline, and tryptophan in 2012, and most amino acids with the exception of asparagine in 2013, and most amino acids in with the exception of glutamic acid when both years were analyzed together (Table 1). ANOVAs also suggested differences in most amino acid levels due to year, with the exception of noninfected levels of glutamic acid, histidine, isoleucine, lysine, and threonine; and Lso-infected levels of alanine, histidine, lysine, proline, threonine, and valine (Table 1).

Regarding differences of amino acid levels between entries, it was clear that interactions between infection status and entry existed. BS2 often had greater amino acid levels than other entries when tubers were noninfected, such as for alanine, glycine, isoleucine, leucine, proline, serine, threonine, and valine (Table 1). However, Atlantic had greater amino acid levels than other entries when tubers were Lso-infected, such as for glycine, histidine, isoleucine, leucine, lysine, methionine, phenylalanine, proline, serine, threonine, and tryptophan (Table 1). Dependent on year and infection status, other patterns existed between entries for different amino acids, as shown in Table 1.

Regarding differences in fold changes due to entries, MANOVA suggested that overall amino acid levels significantly differed between entries for both years $(\Delta=3.199 ; F=3.085 ; P<0.001 ; n=$ 59 for entry; $\Delta=0.953 ; F=30.949 ; P<0.001 ; n=59$ for year; and

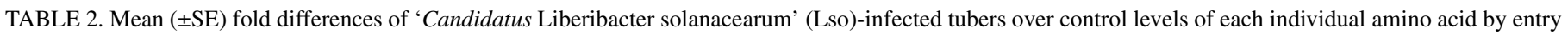
for both years and each year separatelyz

\begin{tabular}{|c|c|c|c|c|c|c|c|c|c|}
\hline \multirow[b]{2}{*}{ Compound } & \multirow[b]{2}{*}{ Year } & \multirow[b]{2}{*}{ Atlantic } & \multirow[b]{2}{*}{ ARS1 } & \multirow[b]{2}{*}{ ARS2 } & \multirow[b]{2}{*}{$\mathrm{BS} 2$} & \multirow[b]{2}{*}{ ZC73 } & \multirow[b]{2}{*}{ ZC74 } & \multicolumn{2}{|c|}{$F$} \\
\hline & & & & & & & & Between entry & Between year \\
\hline \multirow[t]{3}{*}{ Alanine } & Both & $2.19 \pm 0.64$ & $4.36 \pm 2.05$ & $1.40 \pm 0.35$ & $1.92 \pm 0.81$ & $1.35 \pm 0.28$ & $1.84 \pm 0.58$ & 1.59 & $10.133 * *$ \\
\hline & 2012 & $1.05 \pm 0.22 \mathrm{ab}$ & $0.87 \pm 0.15 \mathrm{ab}$ & $0.87 \pm 0.13 \mathrm{ab}$ & $0.78 \pm 0.10 b$ & $1.69 \pm 0.32 \mathrm{a}$ & $0.97 \pm 0.19 \mathrm{ab}$ & $2.745^{*}$ & \\
\hline & 2013 & $3.34 \pm 1.08$ & $7.85 \pm 3.57$ & $2.06 \pm 0.67$ & $3.05 \pm 1.51$ & $1.01 \pm 0.44$ & $2.70 \pm 1.04$ & 1.787 & \\
\hline \multirow[t]{3}{*}{ Asparagine } & Both & $2.14 \pm 0.37 \mathrm{a}$ & $1.27 \pm 0.19 \mathrm{ab}$ & $0.79 \pm 0.14 b$ & $0.83 \pm 0.16 b$ & $1.02 \pm 0.17 \mathrm{~b}$ & $0.66 \pm 0.12 b$ & $6.314 * * *$ & 0.858 \\
\hline & 2012 & $2.20 \pm 0.69$ & $1.11 \pm 0.32$ & $0.93 \pm 0.22$ & $0.93 \pm 0.26$ & $1.24 \pm 0.26$ & $0.76 \pm 0.21$ & 2.003 & \\
\hline & 2013 & $2.08 \pm 0.38 \mathrm{a}$ & $1.42 \pm 0.23 \mathrm{ab}$ & $0.61 \pm 0.15 b$ & $0.73 \pm 0.21 b$ & $0.79 \pm 0.18 b$ & $0.55 \pm 0.10 b$ & $6.903 * * *$ & \\
\hline \multirow[t]{3}{*}{ Aspartic acid } & Both & $5.72 \pm 1.75 \mathrm{a}$ & $1.41 \pm 0.29 b$ & $0.76 \pm 0.12 b$ & $1.00 \pm 0.25 \mathrm{~b}$ & $1.10 \pm 0.18 \mathrm{~b}$ & $1.06 \pm 0.19 \mathrm{~b}$ & $13.980 * * *$ & $18.862 * * *$ \\
\hline & 2012 & $1.60 \pm 0.24 \mathrm{a}$ & $0.78 \pm 0.17 \mathrm{ab}$ & $0.70 \pm 0.17 b$ & $0.67 \pm 0.18 b$ & $1.06 \pm 0.24 \mathrm{ab}$ & $0.78 \pm 0.11 b$ & $3.570 *$ & \\
\hline & 2013 & $9.84 \pm 2.31 \mathrm{a}$ & $2.04 \pm 0.38 \mathrm{~b}$ & $0.84 \pm 0.18 b$ & $1.32 \pm 0.45 b$ & $1.15 \pm 0.29 b$ & $1.34 \pm 0.33 b$ & $11.785 * * *$ & \\
\hline \multirow[t]{3}{*}{ Glutamic acid } & Both & $1.84 \pm 0.56 \mathrm{a}$ & $0.76 \pm 0.12 b$ & $0.56 \pm 0.15 b$ & $0.84 \pm 0.16 b$ & $0.74 \pm 0.16 b$ & $0.82 \pm 0.15 b$ & $5.751 * * *$ & $22.223 * * *$ \\
\hline & 2012 & $3.20 \pm 0.69 \mathrm{a}$ & $0.79 \pm 0.19 b$ & $0.82 \pm 0.18 b$ & $0.98 \pm 0.29 b$ & $0.90 \pm 0.28 b$ & $1.05 \pm 0.15 b$ & $7.275 * * *$ & \\
\hline & 2013 & $0.47 \pm 0.08$ & $0.73 \pm 0.17$ & $0.22 \pm 0.04$ & $0.70 \pm 0.16$ & $0.57 \pm 0.15$ & $0.59 \pm 0.23$ & 1.273 & \\
\hline \multirow[t]{3}{*}{ Glutamine } & Both & $3.65 \pm 1.12 \mathrm{a}$ & $2.74 \pm 0.77 \mathrm{ab}$ & $1.51 \pm 0.27 \mathrm{ab}$ & $2.13 \pm 0.79 a b$ & $1.11 \pm 0.16 b$ & $1.14 \pm 0.20 \mathrm{~b}$ & $3.618 * *$ & $18.144 * * *$ \\
\hline & 2012 & $1.19 \pm 0.18$ & $1.03 \pm 0.04$ & $1.51 \pm 0.35$ & $0.96 \pm 0.05$ & $1.08 \pm 0.07$ & $0.99 \pm 0.17$ & 1.292 & \\
\hline & 2013 & $6.12 \pm 1.59 \mathrm{a}$ & $4.45 \pm 1.11 \mathrm{ab}$ & $1.51 \pm 0.48 \mathrm{ab}$ & $3.31 \pm 1.46 \mathrm{ab}$ & $1.15 \pm 0.34 \mathrm{~b}$ & $1.30 \pm 0.38 b$ & $3.644 *$ & \\
\hline \multirow[t]{3}{*}{ Glycine } & Both & $5.90 \pm 1.72 \mathrm{a}$ & $1.52 \pm 0.29 b$ & $1.01 \pm 0.13 \mathrm{~b}$ & $1.02 \pm 0.12 b$ & $1.10 \pm 0.14 \mathrm{~b}$ & $0.77 \pm 0.09 \mathrm{~b}$ & $26.648 * * *$ & $28.979 * * *$ \\
\hline & 2012 & $1.41 \pm 0.15 \mathrm{a}$ & $0.74 \pm 0.08 \mathrm{~b}$ & $0.82 \pm 0.08 \mathrm{~b}$ & $1.03 \pm 0.11 \mathrm{ab}$ & $1.42 \pm 0.12 \mathrm{a}$ & $0.82 \pm 0.12 b$ & $7.061 * * *$ & \\
\hline & 2013 & $10.39 \pm 1.78 \mathrm{a}$ & $2.30 \pm 0.24 b$ & $1.24 \pm 0.23 b$ & $1.02 \pm 0.24 \mathrm{~b}$ & $0.77 \pm 0.16 b$ & $0.72 \pm 0.13 b$ & $24.427 * * *$ & \\
\hline \multirow[t]{3}{*}{ Histidine } & Both & $12.22 \pm 2.90 \mathrm{a}$ & $2.56 \pm 1.11 \mathrm{~b}$ & $1.62 \pm 0.39 b$ & $2.25 \pm 1.11 \mathrm{~b}$ & $1.29 \pm 0.37 \mathrm{~b}$ & $1.42 \pm 0.37 \mathrm{~b}$ & $13.172 * * *$ & $10.426 * * *$ \\
\hline & 2012 & $6.58 \pm 2.10 \mathrm{a}$ & $0.92 \pm 0.28 b$ & $1.58 \pm 0.59 b$ & $0.88 \pm 0.24 b$ & $1.18 \pm 0.36 b$ & $0.84 \pm 0.14 b$ & $6.080 * * *$ & \\
\hline & 2013 & $17.85 \pm 4.19 a$ & $4.20 \pm 2.03 b$ & $1.68 \pm 0.58 b$ & $3.61 \pm 2.13 b$ & $1.40 \pm 0.68 b$ & $2.01 \pm 0.64 \mathrm{~b}$ & $8.343^{* * * *}$ & \\
\hline Isoleucine & Both & $5.28 \pm 1.30 \mathrm{a}$ & $3.62 \pm 0.95 b$ & $1.83 \pm 0.25 \mathrm{c}$ & $0.93 \pm 0.18 \mathrm{c}$ & $0.90 \pm 0.21 \mathrm{c}$ & $0.76 \pm 0.13 \mathrm{c}$ & $32.539 * * *$ & $56.505 * * *$ \\
\hline & 2012 & $1.61 \pm 0.44$ & $1.18 \pm 0.20$ & $1.59 \pm 0.31$ & $0.79 \pm 0.25$ & $1.42 \pm 0.20$ & $0.77 \pm 0.18$ & 1.863 & \\
\hline & 2013 & $8.94 \pm 0.82 \mathrm{a}$ & $6.06 \pm 1.03 \mathrm{~b}$ & $2.14 \pm 0.40 \mathrm{c}$ & $1.07 \pm 0.27 \mathrm{c}$ & $0.38 \pm 0.14 \mathrm{c}$ & $0.75 \pm 0.22 \mathrm{c}$ & $34.977 * * *$ & \\
\hline Leucine & Both & $3.81 \pm 0.93 \mathrm{a}$ & $2.05 \pm 0.36 \mathrm{~b}$ & $1.40 \pm 0.15 b$ & $0.99 \pm 0.15 b$ & $1.13 \pm 0.15 b$ & $0.81 \pm 0.13 b$ & $14.573 * * *$ & $16.761 * * *$ \\
\hline & 2012 & $1.63 \pm 0.42$ & $1.21 \pm 0.21$ & $1.20 \pm 0.04$ & $0.90 \pm 0.19$ & $1.46 \pm 0.15$ & $0.82 \pm 0.09$ & 2.016 & \\
\hline & 2013 & $5.99 \pm 1.15 \mathrm{a}$ & $2.89 \pm 0.45 b$ & $1.65 \pm 0.30 \mathrm{~b}$ & $1.07 \pm 0.24 \mathrm{~b}$ & $0.79 \pm 0.16 b$ & $0.79 \pm 0.25 b$ & $13.483 * * *$ & \\
\hline Lysine & Both & $13.79 \pm 2.69 \mathrm{a}$ & $4.41 \pm 2.04 \mathrm{~b}$ & $1.50 \pm 0.38 \mathrm{~b}$ & $2.06 \pm 0.79 \mathrm{~b}$ & $0.98 \pm 0.26 \mathrm{~b}$ & $1.85 \pm 0.57 \mathrm{~b}$ & $12.105 * * *$ & $5.960 *$ \\
\hline & 2012 & $11.14 \pm 4.12 \mathrm{a}$ & $0.97 \pm 0.21 b$ & $1.06 \pm 0.38 \mathrm{~b}$ & $1.08 \pm 0.18 b$ & $0.96 \pm 0.33 b$ & $1.00 \pm 0.09 \mathrm{~b}$ & $5.924 * *$ & \\
\hline & 2013 & $16.44 \pm 3.49 \mathrm{a}$ & $7.85 \pm 3.57 \mathrm{ab}$ & $2.06 \pm 0.67 \mathrm{~b}$ & $3.05 \pm 1.51 \mathrm{~b}$ & $1.01 \pm 0.44 \mathrm{~b}$ & $2.70 \pm 1.04 \mathrm{~b}$ & $6.743 * * *$ & \\
\hline Methionine & Both & $7.02 \pm 1.49 \mathrm{a}$ & $1.90 \pm 0.62 b$ & $0.77 \pm 0.16 b$ & $1.43 \pm 0.26 b$ & $0.92 \pm 0.17 b$ & $0.97 \pm 0.17 b$ & $41.444 * * *$ & $40.259 * * *$ \\
\hline & 2012 & $3.03 \pm 0.99 \mathrm{a}$ & $0.47 \pm 0.14 b$ & $0.63 \pm 0.19 b$ & $1.50 \pm 0.39 a b$ & $0.77 \pm 0.23 b$ & $0.77 \pm 0.15 b$ & $4.464 * *$ & \\
\hline & 2013 & $11.02 \pm 1.00 \mathrm{a}$ & $3.32 \pm 0.82 b$ & $0.95 \pm 0.26 b$ & $1.35 \pm 0.39 b$ & $1.07 \pm 0.26 \mathrm{~b}$ & $1.17 \pm 0.28 b$ & $44.082 * * *$ & \\
\hline Phenylalanine & Both & $9.35 \pm 2.43 \mathrm{a}$ & $2.16 \pm 0.44 \mathrm{~b}$ & $2.13 \pm 0.40 \mathrm{~b}$ & $1.53 \pm 0.49 b$ & $0.70 \pm 0.09 \mathrm{~b}$ & $1.34 \pm 0.35 b$ & $40.137 * * *$ & $57.847 * * *$ \\
\hline & 2012 & $2.63 \pm 0.46 \mathrm{a}$ & $1.53 \pm 0.39 \mathrm{ab}$ & $1.60 \pm 0.44 \mathrm{ab}$ & $0.61 \pm 0.18 b$ & $0.69 \pm 0.11 b$ & $0.65 \pm 0.21 b$ & $5.895 * *$ & \\
\hline & 2013 & $16.07 \pm 1.94 \mathrm{a}$ & $2.79 \pm 0.71 b$ & $2.80 \pm 0.62 b$ & $2.44 \pm 0.80 \mathrm{~b}$ & $0.70 \pm 0.16 b$ & $2.03 \pm 0.51 b$ & $34.800 * * *$ & \\
\hline Proline & Both & $12.86 \pm 2.71 \mathrm{a}$ & $3.52 \pm 1.23 \mathrm{~b}$ & $2.72 \pm 1.04 \mathrm{~b}$ & $1.35 \pm 0.50 b$ & $1.77 \pm 0.48 b$ & $1.17 \pm 0.32 \mathrm{~b}$ & $22.602 * * *$ & $14.997 * * *$ \\
\hline & 2012 & $6.66 \pm 2.89 \mathrm{a}$ & $0.33 \pm 0.16 b$ & $3.42 \pm 1.88 \mathrm{ab}$ & $0.53 \pm 0.17 \mathrm{ab}$ & $2.77 \pm 0.71 \mathrm{ab}$ & $0.55 \pm 0.19 a b$ & $2.931 *$ & \\
\hline & 2013 & $19.05 \pm 2.35 \mathrm{a}$ & $6.72 \pm 1.31 \mathrm{~b}$ & $1.83 \pm 0.42 \mathrm{bc}$ & $2.17 \pm 0.88 b c$ & $0.77 \pm 0.18 \mathrm{c}$ & $1.78 \pm 0.47 \mathrm{bc}$ & $33.461 * * *$ & \\
\hline Serine & Both & $4.51 \pm 1.01 \mathrm{a}$ & $1.09 \pm 0.13 b$ & $0.71 \pm 0.07 \mathrm{~b}$ & $1.13 \pm 0.18 b$ & $0.86 \pm 0.15 b$ & $1.16 \pm 0.16 b$ & $26.952 * * *$ & $11.391 * *$ \\
\hline & 2012 & $1.98 \pm 0.48 \mathrm{a}$ & $1.08 \pm 0.24 \mathrm{ab}$ & $0.74 \pm 0.08 b$ & $1.04 \pm 0.21 \mathrm{ab}$ & $1.21 \pm 0.20 \mathrm{ab}$ & $1.09 \pm 0.11 \mathrm{ab}$ & $2.675^{*}$ & \\
\hline & 2013 & $7.04 \pm 1.10 \mathrm{a}$ & $1.10 \pm 0.12 b$ & $0.67 \pm 0.12 b$ & $1.23 \pm 0.31 \mathrm{~b}$ & $0.51 \pm 0.05 b$ & $1.23 \pm 0.31 \mathrm{~b}$ & $25.072 * * *$ & \\
\hline Threonine & Both & $6.35 \pm 1.51 \mathrm{a}$ & $1.50 \pm 0.29 b$ & $0.95 \pm 0.16 b$ & $1.25 \pm 0.26 \mathrm{~b}$ & $0.82 \pm 0.19 b$ & $0.89 \pm 0.10 b$ & $28.947 * * *$ & $18.840 * * *$ \\
\hline & 2012 & $2.59 \pm 0.42 \mathrm{a}$ & $0.78 \pm 0.11 b$ & $0.89 \pm 0.21 b$ & $1.07 \pm 0.21 \mathrm{~b}$ & $1.30 \pm 0.20 b$ & $0.81 \pm 0.10 b$ & $8.868 * * *$ & \\
\hline & 2013 & $10.11 \pm 1.74 \mathrm{a}$ & $2.22 \pm 0.34 \mathrm{~b}$ & $1.02 \pm 0.29 \mathrm{~b}$ & $1.42 \pm 0.49 \mathrm{~b}$ & $0.33 \pm 0.06 b$ & $0.98 \pm 0.19 b$ & $22.428 * * *$ & \\
\hline Tryptophan & Both & $10.71 \pm 4.46 \mathrm{a}$ & $4.59 \pm 1.79 a b$ & $1.19 \pm 0.56 \mathrm{~b}$ & $1.83 \pm 0.53 b$ & $1.57 \pm 0.33 b$ & $1.89 \pm 0.48 b$ & $5.825 * * *$ & $16.351 * * *$ \\
\hline & 2012 & $1.11 \pm 0.14 b$ & $0.88 \pm 0.19 b$ & $0.44 \pm 0.15 b$ & $1.18 \pm 0.35 \mathrm{ab}$ & $2.32 \pm 0.35 \mathrm{a}$ & $0.81 \pm 0.34 \mathrm{~b}$ & $5.676 * *$ & \\
\hline & 2013 & $20.32 \pm 6.60 \mathrm{a}$ & $8.30 \pm 2.75 \mathrm{ab}$ & $2.11 \pm 1.14 \mathrm{~b}$ & $2.48 \pm 0.98 b$ & $0.81 \pm 0.31 b$ & $2.97 \pm 0.60 \mathrm{~b}$ & $5.842 * *$ & \\
\hline Tyrosine & Both & $4.98 \pm 1.34$ & $1.82 \pm 0.58$ & $0.99 \pm 0.16$ & $2.48 \pm 0.87$ & $2.05 \pm 0.75$ & $3.45 \pm 1.66$ & 2.176 & 1.862 \\
\hline & 2012 & $7.15 \pm 1.75 \mathrm{a}$ & $0.54 \pm 0.11 b$ & $0.98 \pm 0.22 b$ & $1.12 \pm 0.26 \mathrm{~b}$ & $1.87 \pm 0.84 \mathrm{~b}$ & $0.92 \pm 0.08 b$ & $9.697 * * *$ & \\
\hline & 2013 & $2.80 \pm 1.61$ & $3.10 \pm 0.82$ & $0.99 \pm 0.27$ & $3.84 \pm 1.56$ & $2.22 \pm 1.35$ & $5.98 \pm 3.04$ & 0.901 & \\
\hline Valine & Both & $3.10 \pm 0.58 \mathrm{a}$ & $4.28 \pm 1.23 \mathrm{a}$ & $1.79 \pm 0.25 b$ & $1.04 \pm 0.19 b$ & $1.18 \pm 0.26 b$ & $0.90 \pm 0.15 b$ & $22.878 * * *$ & $50.955 * * *$ \\
\hline & 2012 & $1.53 \pm 0.32 \mathrm{ab}$ & $0.91 \pm 0.13 b$ & $1.42 \pm 0.12 \mathrm{ab}$ & $0.87 \pm 0.24 \mathrm{~b}$ & $1.86 \pm 0.24 \mathrm{a}$ & $0.74 \pm 0.09 \mathrm{~b}$ & $4.655 * *$ & \\
\hline & 2013 & $4.67 \pm 0.44 b$ & $7.64 \pm 1.06 \mathrm{a}$ & $2.26 \pm 0.46 \mathrm{c}$ & $1.21 \pm 0.30 \mathrm{c}$ & $0.51 \pm 0.13 \mathrm{c}$ & $1.06 \pm 0.29 \mathrm{c}$ & $26.667 * * *$ & \\
\hline
\end{tabular}

z Values over 1 represent greater levels in Lso-infected tubers, whereas values under 1 represent levels greater in noninfected control. $F$ statistics are provided. Different letters represent significant mean separations by Tukey's honest significant difference test. $* P<0.05$; $* * P<0.01$; and $* * * P<0.001$. 
$\Delta=3.106 ; F=2.849 ; P<0.001 ; n=59$ for the entry and year interaction), $2012(\Delta=3.778 ; F=1.889 ; P=0.006 ; n=30)$, and $2013(\Delta=4.381 ; F=3.929 ; P<0.001 ; n=29)$. Levels of most amino acids for both years (with the exception of alanine and tyrosine), 12 amino acids in 2012 (aspartic acid, glutamic acid, glycine, histidine, lysine, methionine, phenylalanine, proline, serine, threonine, tyrosine, and valine) and 15 amino acids in 2013 (asparagine, aspartic acid, glutamine, glycine, histidine, isoleucine, leucine, lysine, methionine, phenylalanine, proline, serine, threonine, tryptophan, and valine) had greater fold increases in Atlantic tubers than tubers from at least one other entry (Table 2$)$. For both years $(F=23.278 ; P<$ $0.001 ; n=59$ for entry; $F=28.905 ; P<0.001 ; n=59$ for year; and $F=8.807 ; P<0.001 ; n=59$ for the entry and year interaction), 2012 $(F=9.600 ; P<0.001 ; n=30)$, and $2013(F=16.533 ; P<0.001 ; n=$ $29)$, the mean fold change of amino acids was significant greater in Atlantic tubers than tubers from all other entries (Fig. 4).

Levels of glycine, isoleucine, leucine, lysine, phenylalanine, proline, serine, threonine, and valine were all positively correlated $(P<0.05)$ with both fresh and fried symptom ratings. Asparagine, aspartic acid, histidine, methionine, and tryptophan were positively correlated $(P<0.05)$ with only fresh symptoms, whereas tyrosine was positively correlated $(P<0.05)$ with only fried symptoms. Lso titers were significantly positively correlated $(P<0.05)$ with asparagine, glutamine, isoleucine, lysine, methionine, phenylalanine, serine, and tryptophan levels.

Sugar responses in susceptible and tolerant entries. Fructose and glucose levels were examined in tuber tissues. Entry

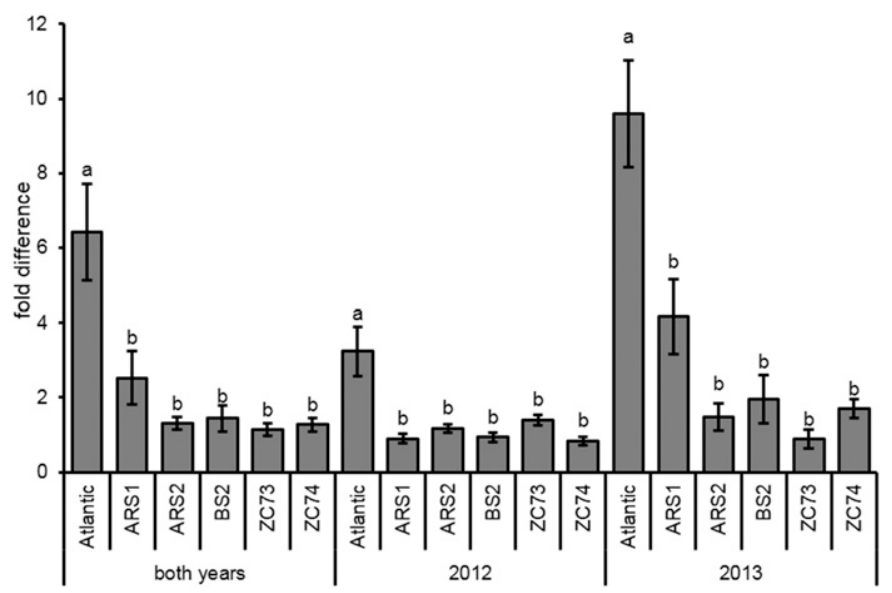

Fig. 4. Fold differences of amino acid levels for 'Candidatus Liberibacter solanacearum'-infected tubers over control tubers of each entry for both years and each year separately. Bars represent standard errors. Different letters represent means that are significantly different by Tukey's honest significant difference test. and infection status significantly affected overall levels of sugars in both years $(\Delta=0.804 ; F=16.927 ; P<0.001 ; n=150$ for entry; $\Delta=$ $0.405 ; F=42.556 ; P<0.001 ; n=150$ for infection status; $\Delta=0.553$; $F=77.363 ; P<0.001 ; n=150$ for year; $\Delta=0.534 ; F=9.186 ; P<$ $0.001 ; n=150$ for the entry and infection status interaction; $\Delta=$ $0.459 ; F=7.513 ; P<0.001 ; n=150$ for the entry and year interaction; $\Delta=0.340 ; F=32.163 ; P<0.001 ; n=150$ for the infection status and year interaction; and $\Delta=0.309 ; F=4.605 ; P<$ $0.001 ; n=150$ for the entry, infection status, and year interaction), $2012(\Delta=0.475 ; F=4.864 ; P<0.001 ; n=90$ for entry; $\Delta=0.119$; $F=5.194 ; P=0.008 ; n=90$ for infection status; and $\Delta=0.301 ; F=$ 2.759; $P=0.004 ; n=90$ for the entry and infection status interaction $)$, and $2013(\Delta=1.113 ; F=12.045 ; P<0.001 ; n=60$ for entry; $\Delta=0.565 ; F=30.476 ; P<0.001 ; n=60$ for infection status; and $\Delta=0.888 ; F=7.592 ; P<0.001 ; n=60$ for the entry and infection status interaction). Because MANOVAs suggested significant differences due to year, entry, and infection interactions, ANOVAs were repeated with treatment combinations separated out. ANOVAs suggested significant $(P<0.05)$ effects due to treatment combination for both sugars and both years, 2012, and 2013. For both years, tubers from the BS2 entry had consistently greater amounts of sugars than all other cultivars, except Atlantic regardless of infection status (Table 3). Infected Atlantic tubers had similar fructose levels to infected BS2 tubers, but noninfected BS2 had greater levels of both fructose and glucose than Atlantic tubers (Table 3). For 2012, infected Atlantic tuber levels of fructose were greater than other entry levels (Table 3). However, fructose and glucose levels did not differ between entries when tubers were not infected (Table 3). For 2013, infected BS2 had greater levels of fructose than all other combinations except infected Atlantic tubers, and glucose levels were greater in infected BS2 than all other treatment combinations (Table 3). BS2 levels of fructose and glucose were greater than most other entries when noninfected, with the exception of no significant difference between BS2 and ZC73 fructose levels (Table 3).

Regarding variations in fold increases between entries, MANOVA suggested significant differences between entries for both years $(\Delta=0.589 ; F=4.008 ; P<0.001 ; n=60$ for entry; $\Delta=0.266$; $F=8.496 ; P<0.001 ; n=60$ for year; and $\Delta=0.669 ; F=4.824 ; P<$ $0.001 ; n=60$ for the entry and year interaction), $2012(\Delta=0.670$; $F=2.415 ; P=0.020 ; n=30)$, and $2013(\Delta=1.091 ; F=5.762 ; P<$ $0.001 ; n=30)$. For fructose fold changes were not significantly different among entries for $2012(F=1.817 ; P=0.148 ; n=30)$, but were significant for $2013(F=4.982 ; P=0.003 ; n=30)$ and when both years were analyzed together $(F=5.642 ; P<0.001 ; n=60$ for entry; $F=16.854 ; P<0.001 ; n=60$ for year; and $F=4.003$; $P<0.001 ; n=60$ for the entry and year interaction). Greater differences were observed in Atlantic than ARS1, BS2, ZC73, and ZC74 in 2013 (Fig. 5), and in Atlantic compared with ARS1 and ZC73 when both years were analyzed together. Likewise, for glucose

TABLE 3. Mean $( \pm \mathrm{SE})$ sugar levels $(\mu \mathrm{g} / \mathrm{g} \mathrm{FW})$ separated by entry for both years and each year separately ${ }^{\mathrm{z}}$

\begin{tabular}{|c|c|c|c|c|c|c|c|c|c|c|c|}
\hline \multirow[b]{2}{*}{ Compound } & \multirow[b]{2}{*}{ Year } & \multirow[b]{2}{*}{ Treatment } & \multirow[b]{2}{*}{ Atlantic } & \multirow[b]{2}{*}{ ARS1 } & \multirow[b]{2}{*}{ ARS2 } & \multirow[b]{2}{*}{ BS2 } & \multirow[b]{2}{*}{ ZC73 } & \multirow[b]{2}{*}{ ZC74 } & \multicolumn{3}{|c|}{$F$} \\
\hline & & & & & & & & & $\begin{array}{l}\text { Within } \\
\text { treatment }\end{array}$ & $\begin{array}{c}\text { Between } \\
\text { year }\end{array}$ & $\begin{array}{c}\text { Between } \\
\text { treatment }\end{array}$ \\
\hline \multirow[t]{6}{*}{ Fructose } & \multirow[t]{2}{*}{ Both } & Control & $262 \pm 67 b$ & $306 \pm 74 b$ & $200 \pm 58 b$ & $917 \pm 337 \mathrm{a}$ & $223 \pm 84 b$ & $138 \pm 34 b$ & $5.572 * * *$ & $6.338 *$ & \multirow[t]{2}{*}{$68.044 * * *$} \\
\hline & & Lso+ & $1800 \pm 280 \mathrm{a}$ & $308 \pm 57 \mathrm{~b}$ & $783 \pm 222 b$ & $1490 \pm 360 a$ & $319 \pm 95 b$ & $327 \pm 102 b$ & $31.015 * * *$ & $99.349 * * *$ & \\
\hline & \multirow{2}{*}{2012} & Control & $321 \pm 114$ & $266 \pm 116$ & $241 \pm 104$ & $353 \pm 63$ & $43 \pm 6$ & $86 \pm 39$ & 2.239 & & \multirow[t]{2}{*}{$10.484 * *$} \\
\hline & & Lso+ & $1310 \pm 260 a$ & $222 \pm 67 b c$ & $261 \pm 82 b c$ & $646 \pm 120 b$ & $178 \pm 65 \mathrm{bc}$ & $60 \pm 9 c$ & $13.403 * * *$ & & \\
\hline & \multirow[t]{2}{*}{2013} & Control & $190 \pm 54 \mathrm{~b}$ & $354 \pm 97 b$ & $150 \pm 29 \mathrm{~b}$ & $1590 \pm 640 a$ & $438 \pm 133 a b$ & $200 \pm 49 b$ & $4.163 * *$ & & \multirow[t]{2}{*}{$42.941 * * *$} \\
\hline & & Lso+ & $2690 \pm 390 \mathrm{ab}$ & $462 \pm 65 d$ & $1720 \pm 280 b c$ & $3000 \pm 460 a$ & $572 \pm 206 \mathrm{~cd}$ & $806 \pm 70 \mathrm{~cd}$ & $14.959 * * *$ & & \\
\hline \multirow[t]{6}{*}{ Glucose } & \multirow[t]{2}{*}{ Both } & Control & $260 \pm 87 \mathrm{~b}$ & $142 \pm 37 \mathrm{~b}$ & $190 \pm 62 \mathrm{~b}$ & $1390 \pm 51 \mathrm{a}$ & $220 \pm 76 b$ & $505 \pm 202 b$ & $6.973^{* * *} *$ & $15.122 * * *$ & \multirow[t]{2}{*}{$80.768^{* * *}$} \\
\hline & & Lso+ & $1620 \pm 400 \mathrm{~b}$ & $1040 \pm 390 \mathrm{bc}$ & $1340 \pm 450 \mathrm{bc}$ & $2680 \pm 910 a$ & $413 \pm 139 c$ & $752 \pm 274 \mathrm{bc}$ & $16.087 * * *$ & $176.145^{* * *}$ & \\
\hline & \multirow[t]{2}{*}{2012} & Control & $44.5 \pm 30.7$ & $48.6 \pm 18.4$ & $28.7 \pm 14.6$ & $436 \pm 348$ & $21.9 \pm 9.2$ & $460 \pm 376$ & 1.034 & & \multirow[t]{2}{*}{1.539} \\
\hline & & Lso+ & $790 \pm 231 \mathrm{a}$ & $21.0 \pm 4.0 \mathrm{~b}$ & $272 \pm 246 a b$ & $564 \pm 185 a b$ & $195 \pm 166 a b$ & $20.1 \pm 3.9 b$ & $3.254 *$ & & \\
\hline & \multirow[t]{2}{*}{2013} & Control & $518 \pm 98 b$ & $254 \pm 35 \mathrm{~b}$ & $384 \pm 60 \mathrm{~b}$ & $2530 \pm 820 \mathrm{a}$ & $458 \pm 74 b$ & $558 \pm 102 b$ & $6.443 * * *$ & & \multirow[t]{2}{*}{$61.740 * * *$} \\
\hline & & Lso+ & $3100 \pm 610 b$ & $2880 \pm 31 b$ & $3270 \pm 45 b$ & $6490 \pm 1320 a$ & $806 \pm 132 b$ & $2070 \pm 80 \mathrm{~b}$ & $8.815^{* * * *}$ & & \\
\hline
\end{tabular}

${ }^{\mathrm{z}} F$ statistics are provided. Different letters represent significant mean separations by Tukey's honest significant difference test. $* P<0.05 ; * * P<0.01 ;$ and $* * * P<0.001$. 
fold changes were significantly different among entries for both years $(F=6.623 ; P<0.001 ; n=30$ for entry; $F=0.294 ; P=0.590 ; n=60$ for year; and $F=5.699 ; P<0.001 ; n=60$ for the entry and year interaction), $2012(F=6.174 ; P<0.001 ; n=30)$, and $2013(F=6.090$; $P=0.001 ; n=30)$, with greater differences in Atlantic than ARS2, BS2, ZC73, and ZC74 when both years analyzed together; greater differences in Atlantic than all other entries in 2012; and greater differences in ARS1 and ARS2 than ZC73 in 2013 (Fig. 5).

Fructose and glucose levels were significantly positively correlated $(P<0.05)$ with fresh symptoms, fried symptoms, and Lso titers.

Phenolic responses in susceptible and tolerant entries. A total of 12 phenolics were quantified including chlorogenic acids (chlorogenic acid, a chlorogenic acid derivative, cryptochlorogenic acid, and a cryptochlorogenic acid derivative), flavonoids (four flavonoid glycoside derivatives and one flavonoid methyl ester glycoside), and phenolic acids (protocatechuic acid glycoside and a quinic acid derivative).

Entry and infection status significantly affected overall levels of phenolics in both years $(\Delta=2.023 ; F=6.739 ; P<0.001 ; n=150$ for entry; $\Delta=0.710 ; F=23.411 ; P<0.001 ; n=150$ for infection status; $\Delta=0.747 ; F=28.314 ; P<0.001 ; n=150$ for year; $\Delta=1.648 ; F=$ 4.875; $P<0.001 ; n=150$ for the entry and infection status interaction; $\Delta=2.052 ; F=6.901 ; P<0.001 ; n=150$ for the entry and year interaction; $\Delta=0.610 ; F=14.986 ; P<0.001 ; n=150$ for the infection status and year interaction; and $\Delta=1.434 ; F=3.988$; $P<0.001 ; n=150$ for the entry, infection status, and year interaction), $2012(\Delta=2.514 ; F=6.621 ; P<0.001 ; n=90$ for entry; $\Delta=0.778 ; F=21.668 ; P<0.001 ; n=90$ for infection status; and $\Delta=1.659 ; F=3.250 ; P<0.001 ; n=90$ for the entry and infection status interaction $)$, and $2013(\Delta=3.225 ; F=6.939 ; P<0.001 ; n=60$ for entry; $\Delta=0.941 ; F=55.231 ; P<0.001 ; n=60$ for infection status; and $\Delta=3.016 ; F=5.806 ; P<0.001 ; n=60$ for the entry and

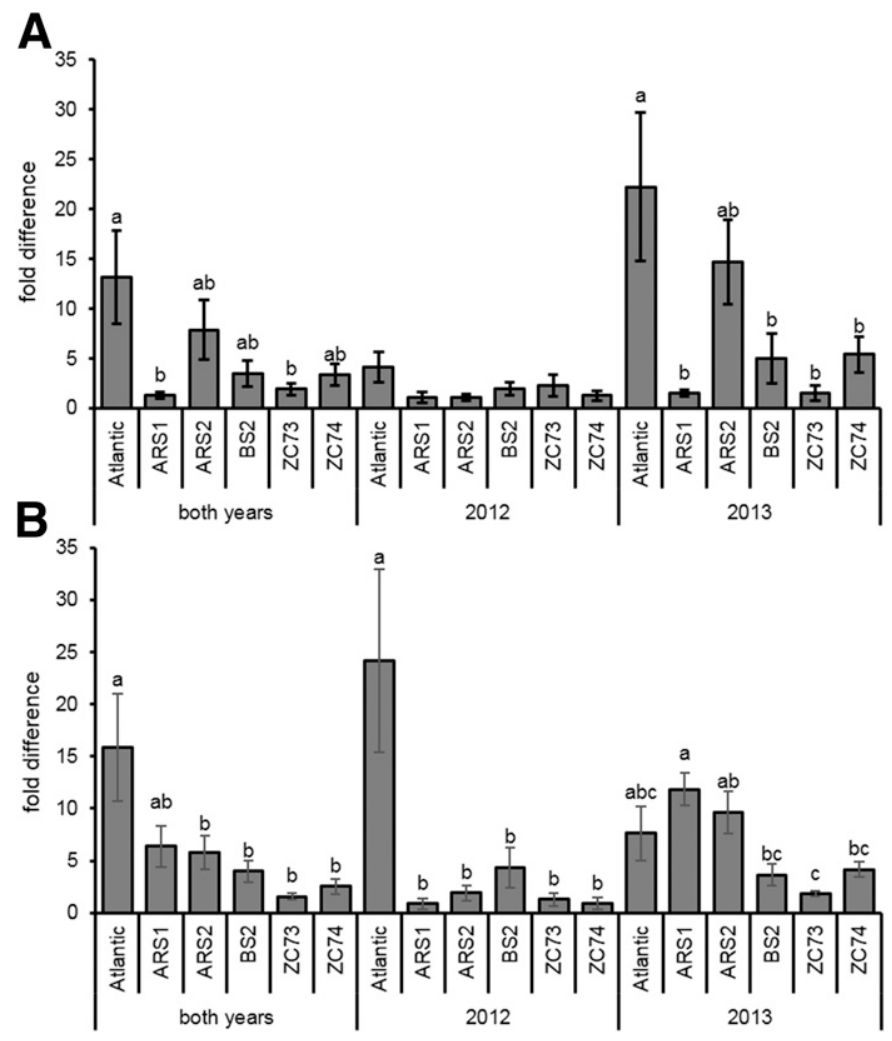

Fig. 5. Fold differences of $\mathbf{A}$, fructose or $\mathbf{B}$, glucose levels for 'Candidatus Liberibacter solanacearum'-infected tubers over control tubers of each entry for both years and each year separately. Bars represent standard errors. Different letters represent means that are significantly different by Tukey's honest significant difference test. infection status interaction). Because of significant interactions, ANOVAs were repeated with treatment combinations separated out.

For both years and each year individually, all compounds were significantly different due to infection status with greater phenolic compound levels present in Lso-infected than noninfected tubers (Table 4). With a few exceptions, such as for flavonoid glycoside 1 and quinic acid derivative levels in 2013 only, Atlantic tubers that were Lso-infected had greater phenolic compound levels than Lsoinfected tubers from the other entries (Table 4). Differences between entries when tubers were noninfected were much more variable and dependent on the particular phenolic compound analyzed (Table 4). However, noninfected BS2 tubers had greater levels of chlorogenic acid, the chlorogenic acid derivative, and protocatechuic acid glycoside when both years were analyzed as well as for each year individually (Table 4).

Regarding differences in fold changes due to entries, MANOVA suggested that overall phenolic levels significantly differed between entries for both years $(\Delta=2.043 ; F=2.637 ; P<0.001 ; n=60$ for entry; $\Delta=0.709 ; F=8.415 ; P<0.001 ; n=60$ for year; and $\Delta=1.932 ; F=2.404 ; P<0.001 ; n=60$ for the entry and year interaction), $2012(\Delta=2.708 ; F=1.934 ; P=0.003 ; n=30)$, and $2013(\Delta=3.502 ; F=3.313 ; P<0.001 ; n=30)$. When both years were analyzed together, almost every phenolic compound, with the exception of flavonoid glycoside 1, was greater in Atlantic in fold change than the ARS1, ARS2, ZC73, and ZC74 entries (Table 5). Levels of all analyzed phenolics in 2012 and all but three (a cryptochlorogenic acid derivate, flavonoid glycoside 1, and a quinic acid derivative) in 2013 had greater fold increases in Atlantic tubers than tubers from at least one other entry (Table 5). For both years $(F=60.481 ; P<0.001 ; n=60$ for entry; $F=12.048 ; P<0.001$; $n=60$ for year; and $F=2.089 ; P=0.083 ; n=60$ for the entry and year interaction), $2012(F=20.169 ; P<0.001 ; n=30)$, and 2013 $(F=49.460 ; P<0.001 ; n=29)$, the mean fold change of phenolic compounds was significantly greater in Atlantic tubers than tubers from all other entries (Fig. 6).

The levels of all analyzed individual phenolics except the flavonoid methyl ester glycoside were significantly positively correlated $(P<$ 0.05 ) with both fresh and fried symptoms. The flavonoid methyl ester was significantly positively correlated $(P<0.05)$ with only fresh symptoms. Lso titers were significantly positively correlated $(P<0.05)$ with levels of chlorogenic acid, cryptochlorogenic acid, cryptochlorogenic acid derivative, flavonoid glycoside 2 , and protocatechuic acid glycoside.

\section{DISCUSSION}

After multiple years of screening for tolerance, a total of five potato advanced breeding clones exhibited little to no fresh and fried ZC symptoms when infected with Lso. Lso titers were also significantly reduced for at least some of the breeding clones relative to susceptible Atlantic, albeit only marginally statistically so in 2013.

Year-to-year variations were likely attributed to uncontrolled climatic factors which would affect vectoring efficacy and well as infection progression and tuber development. Indeed, much more variability in pathogen titers occurred in 2013 overall, but generally titers were much greater in that year, suggesting more favorable conditions for Lso infections to progress than 2012. Furthermore, climate likely influenced differences in symptoms and physiology between years, with two cultivars, BS2 in 2012 and ARS2 in 2013, having no statistical difference in evaluation of fried slices taken at the time of chemical analyses in one of the two years (albeit both did consistent differ when a larger number of fried slices were evaluated upon harvest). Greater tuber levels for many of the observed compounds were present in Lso-infected tubers overall in 2013, implying, with generally greater Lso titers, that infections proceeded further than in 2012 as well.

However, despite variations between years, overall findings demonstrate that Lso tolerance and possible resistance to Lso likely 
exists in wild species of potato, and can be exploited to create commercially viable, ZC-resistant cultivars. Additional breeding clones are being screened to discover even more possibly Lsoinfection tolerant potato lines.

Unique to this study is an examination of host chemistry in an effort to find a potential mechanism of tolerance. Regarding differences in noninfected, constitutive (baseline) levels of metabolites between entries, few if any relationships to ZC progression and Lso titers were observed. Indeed, the majority of differences between entries in noninfected tubers where greater levels of certain amino acids, sugars, and phenolics in BS2 compared with all other entries (including the susceptible Atlantic and putative tolerant varieties). This was likely the result of BS2 being bred to be a red marketing type of potato, whereas the others were chipping marketing types. Regardless, because of few differences in the physiology of Atlantic from the tested breeding lines, it seems unlikely that physiological markers of tolerance could be used prior to actual infection by Lso.

TABLE 4. Mean $\left( \pm\right.$ SE) phenolic compound levels $(\mu \mathrm{g} / \mathrm{g} \mathrm{FW})$ separated by entry for both years and each year separately ${ }^{\mathrm{z}}$

\begin{tabular}{|c|c|c|c|c|c|c|c|c|c|c|c|}
\hline \multirow[b]{2}{*}{ Compound } & \multirow[b]{2}{*}{ Year } & \multirow[b]{2}{*}{ Treatment } & \multirow[b]{2}{*}{ Atlantic } & \multirow[b]{2}{*}{ ARS1 } & & & & & & $F$ & \\
\hline & & & & & ARS2 & BS2 & ZC73 & ZC74 & $\begin{array}{c}\text { Within } \\
\text { treatment }\end{array}$ & $\begin{array}{l}\text { Between } \\
\text { year }\end{array}$ & $\begin{array}{l}\text { Between } \\
\text { treatment }\end{array}$ \\
\hline Chlorogenic acid & Both & $\begin{array}{l}\text { Control } \\
\text { Lso+ }\end{array}$ & $\begin{array}{l}4.58 \pm 1.09 \mathrm{bc} \\
479 \pm 82 \mathrm{a}\end{array}$ & $\begin{array}{l}2.42 \pm 0.24 \mathrm{c} \\
29.0 \pm 4.1 \mathrm{~b}\end{array}$ & $\begin{array}{c}8.42 \pm 2.18 \mathrm{abc} \\
50.6 \pm 9.2 \mathrm{~b}\end{array}$ & $\begin{array}{l}13.2 \pm 3.2 \mathrm{a} \\
66.9 \pm 12.6 \mathrm{~b}\end{array}$ & $\begin{array}{l}6.67 \pm 2.14 b c \\
17.8 \pm 3.6 b\end{array}$ & $\begin{array}{l}10.6 \pm 2.5 \mathrm{ab} \\
30.3 \pm 4.6 \mathrm{~b}\end{array}$ & $\begin{array}{r}6.454^{* * * *} \\
47.046 * * *\end{array}$ & $\begin{array}{l}56.472 * * * \\
28.432 * * *\end{array}$ & $81.091 * * *$ \\
\hline & 2012 & Control & $5.89 \pm 1.88 \mathrm{bc}$ & $2.92 \pm 0.21 \mathrm{c}$ & $13.3 \pm 2.6 \mathrm{abc}$ & $20.5 \pm 3.8 \mathrm{a}$ & $11.1 \pm 2.9 \mathrm{abc}$ & $16.6 \pm 2.7 \mathrm{ab}$ & $6.400^{* * *}$ & & $69.520 * * *$ \\
\hline & & Lso+ & $657 \pm 76 \mathrm{a}$ & $21.5 \pm 4.1 \mathrm{~b}$ & $70.8 \pm 8.3 \mathrm{~b}$ & $85.2 \pm 16.3 \mathrm{~b}$ & $26.2 \pm 2.7 \mathrm{~b}$ & $20.4 \pm 2.3 \mathrm{~b}$ & $61.881^{* * * *}$ & & \\
\hline & 2013 & Control & $3.01 \pm 0.26 \mathrm{ab}$ & $1.81 \pm 0.30 \mathrm{~b}$ & $2.54 \pm 0.42 \mathrm{ab}$ & $4.52 \pm 0.80 \mathrm{a}$ & $1.38 \pm 0.34 \mathrm{~b}$ & $3.45 \pm 0.98 \mathrm{ab}$ & $3.789^{*}$ & & $104.081 * * *$ \\
\hline & & Lso+ & $159 \pm 25 \mathrm{a}$ & $42.6 \pm 4.5 \mathrm{~b}$ & $14.2 \pm 1.8 \mathrm{~b}$ & $34.0 \pm 8.2 \mathrm{~b}$ & $2.75 \pm 0.40 \mathrm{~b}$ & $48.1 \pm 7.2 \mathrm{~b}$ & $24.407 * * *$ & & \\
\hline Chlorogenic acid & & & & & & & & & & & \\
\hline derivative & Both & Control & $7.65 \pm 2.32 \mathrm{bc}$ & $5.28 \pm 1.43 \mathrm{c}$ & $10.7 \pm 2.6 \mathrm{bc}$ & $21.7 \pm 5.7 \mathrm{a}$ & $3.76 \pm 1.08 \mathrm{c}$ & $14.1 \pm 3.7 \mathrm{~b}$ & $13.060^{* * * *}$ & $121.779 * * *$ & $126.776 * * *$ \\
\hline & & Lso+ & $389 \pm 59 \mathrm{a}$ & $26.0 \pm 3.2 \mathrm{c}$ & $39.6 \pm 7.3 \mathrm{bc}$ & $93.9 \pm 19.2 \mathrm{~b}$ & $18.6 \pm 4.1 \mathrm{c}$ & $31.4 \pm 3.4 \mathrm{c}$ & $69.234 * * *$ & $60.387 * * *$ & \\
\hline & 2012 & Control & $13.2 \pm 2.5 \mathrm{bc}$ & $9.17 \pm 0.90 \mathrm{c}$ & $17.3 \pm 2.4 \mathrm{bc}$ & $35.5 \pm 5.9 \mathrm{a}$ & $6.08 \pm 1.37 \mathrm{c}$ & $23.9 \pm 2.4 \mathrm{ab}$ & $12.615^{* * * *}$ & & $135.768 * * *$ \\
\hline & & Lso+ & $526 \pm 42 \mathrm{a}$ & $23.4 \pm 3.7 \mathrm{c}$ & $56.3 \pm 5.9 \mathrm{bc}$ & $130 \pm 22 b$ & $28.2 \pm 3.0 \mathrm{c}$ & $37.0 \pm 2.1 \mathrm{c}$ & $100.785^{* * *}$ & & \\
\hline & 2013 & Control & $1.06 \pm 0.23 \mathrm{~b}$ & $0.600 \pm 0.101 \mathrm{~b}$ & $2.66 \pm 0.42 \mathrm{ab}$ & $5.11 \pm 0.72 \mathrm{a}$ & $0.978 \pm 0.307 \mathrm{~b}$ & $2.18 \pm 1.51 \mathrm{ab}$ & $5.347^{* *}$ & & $25.242 * * * *$ \\
\hline & & Lso+ & $143 \pm 43 \mathrm{a}$ & $30.7 \pm 5.9 \mathrm{~b}$ & $9.50 \pm 2.82 \mathrm{~b}$ & $29.6 \pm 6.6 \mathrm{~b}$ & $1.31 \pm 0.19 \mathrm{~b}$ & $21.2 \pm 6.9 \mathrm{~b}$ & $8.307^{* * * *}$ & & \\
\hline Cryptochlorogenic & & & & & & & & & & & \\
\hline acid & Both & Control & $1.68 \pm 0.15 \mathrm{~b}$ & $2.44 \pm 0.30 \mathrm{ab}$ & $3.53 \pm 0.25 a b$ & $5.58 \pm 0.91 \mathrm{a}$ & $2.76 \pm 0.24 \mathrm{ab}$ & $5.04 \pm 1.53 \mathrm{a}$ & $3.864 * *$ & 0.435 & 104.081 *** \\
\hline & & Lso+ & $220 \pm 31 \mathrm{a}$ & $26.0 \pm 7.1 \mathrm{~b}$ & $22.0 \pm 2.9 \mathrm{~b}$ & $39.6 \pm 6.7 \mathrm{~b}$ & $9.12 \pm 1.85 \mathrm{~b}$ & $24.4 \pm 5.3 \mathrm{~b}$ & $30.516^{* * * *}$ & 1.565 & \\
\hline & 2012 & Control & $1.72 \pm 0.18 \mathrm{~b}$ & $3.21 \pm 0.19 \mathrm{ab}$ & $3.13 \pm 0.34 \mathrm{ab}$ & $5.51 \pm 1.64 \mathrm{a}$ & $2.90 \pm 0.19 a b$ & $5.71 \pm 1.00 \mathrm{a}$ & $3.814 * *$ & & $33.045 * * *$ \\
\hline & & Lso+ & $229 \pm 40 \mathrm{a}$ & $8.20 \pm 1.79 \mathrm{~b}$ & $20.6 \pm 3.8 \mathrm{~b}$ & $26.4 \pm 4.6 \mathrm{~b}$ & $12.2 \pm 2.3 \mathrm{~b}$ & $13.9 \pm 1.8 \mathrm{~b}$ & $27.857 * * *$ & & \\
\hline & 2013 & Control & $1.63 \pm 0.26$ & $1.51 \pm 0.20$ & $4.01 \pm 0.26$ & $5.66 \pm 0.75$ & $2.59 \pm 0.49$ & $4.24 \pm 3.32$ & 1.344 & & $41.744 * * *$ \\
\hline & & Lso+ & $204 \pm 56 \mathrm{a}$ & $58.0 \pm 6.8 \mathrm{~b}$ & $24.4 \pm 4.9 \mathrm{~b}$ & $63.2 \pm 10.7 \mathrm{~b}$ & $3.66 \pm 0.36 \mathrm{~b}$ & $43.3 \pm 10.5 b$ & $8.941^{* * *}$ & & \\
\hline Cryptochlorogenic & & & & & & & & & & & \\
\hline acid derivative & Both & Control & $1.80 \pm 0.36$ & $2.54 \pm 0.49$ & $1.08 \pm 0.11$ & $2.68 \pm 1.66$ & $1.42 \pm 0.25$ & $7.19 \pm 4.02$ & 2.243 & 2.332 & $25.117 * * *$ \\
\hline & & Lso+ & $168 \pm 51 \mathrm{a}$ & $38.8 \pm 14.0 \mathrm{~b}$ & $13.5 \pm 3.3 \mathrm{~b}$ & $32.8 \pm 13.1 \mathrm{~b}$ & $5.36 \pm 2.44 \mathrm{~b}$ & $33.9 \pm 12.9 \mathrm{~b}$ & $5.499^{* * * *}$ & 2.06 & \\
\hline & 2012 & Control & $0.820 \pm 0.146$ & $1.74 \pm 0.16$ & $1.21 \pm 0.11$ & $4.08 \pm 3.03$ & $1.96 \pm 0.29$ & $1.28 \pm 0.15$ & 0.868 & & $6.098^{*}$ \\
\hline & & Lso+ & $217 \pm 74 \mathrm{a}$ & $2.41 \pm 0.64 \mathrm{~b}$ & $5.52 \pm 1.04 \mathrm{~b}$ & $1.59 \pm 0.46 \mathrm{~b}$ & $8.18 \pm 3.50 b$ & $3.40 \pm 2.06 \mathrm{~b}$ & $8.253^{* * *}$ & & \\
\hline & 2013 & Control & $2.98 \pm 0.21$ & $3.51 \pm 0.92$ & $0.919 \pm 0.191$ & $1.01 \pm 0.16$ & $0.776 \pm 0.173$ & $14.3 \pm 8.1$ & 2.442 & & $68.330^{* * *}$ \\
\hline & & Lso+ & $79.5 \pm 33.5 \mathrm{a}$ & $104 \pm 10 \mathrm{a}$ & $28.0 \pm 3.6 \mathrm{ab}$ & $89.0 \pm 18.3 \mathrm{a}$ & $0.274 \pm 0.075 b$ & $88.8 \pm 17.9 \mathrm{a}$ & $5.348^{* * *}$ & & \\
\hline Flavonoid & & & & & & & & & & & \\
\hline glycoside 1 & Both & Control & $5.48 \pm 2.73 \mathrm{~b}$ & $2.67 \pm 0.73 \mathrm{~b}$ & $1.77 \pm 0.85 \mathrm{~b}$ & $15.1 \pm 5.0 \mathrm{a}$ & $6.13 \pm 1.71 \mathrm{~b}$ & $9.27 \pm 3.32 \mathrm{ab}$ & $4.663^{* * *}$ & $35.239 * * *$ & $72.224 * * *$ \\
\hline & & Lso+ & $35.2 \pm 5.7 \mathrm{a}$ & $19.9 \pm 4.1 \mathrm{bc}$ & $11.2 \pm 1.8 \mathrm{~cd}$ & $24.7 \pm 2.5 \mathrm{~b}$ & $9.27 \pm 2.55 \mathrm{~d}$ & $8.77 \pm 1.33 \mathrm{~d}$ & $15.723 * * *$ & 2.263 & \\
\hline & 2012 & Control & $9.73 \pm 4.40 \mathrm{~b}$ & $4.39 \pm 0.75 b$ & $2.43 \pm 1.48 \mathrm{~b}$ & $26.8 \pm 5.6 \mathrm{a}$ & $10.9 \pm 0.9 \mathrm{ab}$ & $13.9 \pm 5.3 \mathrm{ab}$ & $5.453^{* *}$ & & $14.482 * * *$ \\
\hline & & Lso+ & $45.9 \pm 6.2 \mathrm{a}$ & $9.59 \pm 1.73 \mathrm{c}$ & $8.08 \pm 1.77 \mathrm{c}$ & $27.9 \pm 2.9 \mathrm{~b}$ & $13.9 \pm 3.0 \mathrm{c}$ & $10.1 \pm 1.9 \mathrm{c}$ & $20.365^{* * * *}$ & & \\
\hline & 2013 & Control & $0.391 \pm 0.080$ & $0.601 \pm 0.386$ & $0.973 \pm 0.644$ & $1.08 \pm 0.17$ & $0.453 \pm 0.205$ & $3.71 \pm 2.09$ & 1.904 & & $183.605 * * *$ \\
\hline & & Lso+ & $15.8 \pm 3.6 \mathrm{bc}$ & $38.5 \pm 2.9 \mathrm{a}$ & $16.8 \pm 2.2 \mathrm{bc}$ & $19.0 \pm 3.4 \mathrm{~b}$ & $0.953 \pm 0.515 \mathrm{~d}$ & $6.44 \pm 1.25 \mathrm{~cd}$ & $25.391 * * *$ & & \\
\hline Flavonoid & & & & & & & & & & & \\
\hline glycoside 2 & Both & $\begin{array}{l}\text { Control } \\
\text { Lso+ }\end{array}$ & $\begin{array}{c}0.181 \pm 0.039 \mathrm{~b} \\
19.8 \pm 5.6 \mathrm{a}\end{array}$ & $\begin{array}{l}0.505 \pm 0.143 \mathrm{a} \\
3.99 \pm 1.77 \mathrm{~b}\end{array}$ & $\begin{array}{l}0.339 \pm 0.068 \mathrm{ab} \\
1.06 \pm 0.26 \mathrm{~b}\end{array}$ & $\begin{array}{l}0.113 \pm 0.029 b \\
1.81 \pm 0.60 b\end{array}$ & $0.292 \pm 0.079 \mathrm{ab}$ & $\begin{array}{l}0.320 \pm 0.070 a b \\
1.32 \pm 0.22 b\end{array}$ & $\begin{array}{l}6.440 * * * \\
7942 * * *\end{array}$ & $\begin{array}{l}1.314 \\
0.553\end{array}$ & $15.075 * * *$ \\
\hline & 2012 & $\begin{array}{l}\text { Lso+ } \\
\text { Control }\end{array}$ & $\begin{array}{l}19.8 \pm 5.6 \mathrm{a} \\
0.232 \pm 0.061 \mathrm{a}\end{array}$ & $\begin{array}{l}3.99 \pm 1.77 \mathrm{~b} \\
0.171 \pm 0.029 \mathrm{a}\end{array}$ & $\begin{array}{l}1.06 \pm 0.26 \mathrm{~b} \\
0.503 \pm 0.067 \mathrm{a}\end{array}$ & $\begin{array}{l}1.81 \pm 0.60 \mathrm{~b} \\
0.176 \pm 0.035 \mathrm{a}\end{array}$ & $\begin{array}{l}0.391 \pm 0.068 \mathrm{~b} \\
0.431 \pm 0.118 \mathrm{a}\end{array}$ & $\begin{array}{l}1.32 \pm 0.22 \mathrm{~b} \\
0.387 \pm 0.120 \mathrm{a}\end{array}$ & $\begin{array}{l}7.942^{* * * *} \\
3.124^{*}\end{array}$ & & $8.592 * *$ \\
\hline & & Lso+ & $24.9 \pm 8.1 \mathrm{a}$ & $0.454 \pm 0.113 \mathrm{~b}$ & $1.48 \pm 0.33 \mathrm{~b}$ & $2.50 \pm 0.85 b$ & $0.530 \pm 0.066 \mathrm{~b}$ & $1.65 \pm 0.28 b$ & $8.426^{* * *}$ & & \\
\hline & 2013 & Control & $0.119 \pm 0.036 b$ & $0.906 \pm 0.195 \mathrm{a}$ & $0.143 \pm 0.030 \mathrm{~b}$ & $0.0366 \pm 0.0071 \mathrm{~b}$ & $0.126 \pm 0.024 \mathrm{~b}$ & $0.240 \pm 0.043 \mathrm{~b}$ & $14.562 * * *$ & & $16.865 * * *$ \\
\hline & & Lso+ & $10.7 \pm 3.7 \mathrm{a}$ & $10.4 \pm 3.6 \mathrm{a}$ & $0.315 \pm 0.078 b$ & $0.564 \pm 0.276 \mathrm{~b}$ & $0.139 \pm 0.034 \mathrm{~b}$ & $0.708 \pm 0.144 \mathrm{~b}$ & $6.134 * * *$ & & \\
\hline Flavonoid & & & & & & & & & & & \\
\hline glycoside 3 & Both & Control & $0.246 \pm 0.041 \mathrm{~b}$ & $0.374 \pm 0.040 \mathrm{~b}$ & $1.42 \pm 0.35 \mathrm{a}$ & $0.241 \pm 0.060 \mathrm{~b}$ & $0.609 \pm 0.210 \mathrm{~b}$ & $0.500 \pm 0.113 \mathrm{~b}$ & $9.955^{* * *}$ & $15.088 * * *$ & $61.798 * * *$ \\
\hline & & Lso+ & $13.3 \pm 3.0 \mathrm{a}$ & $3.68 \pm 0.42 \mathrm{~b}$ & $6.04 \pm 1.19 \mathrm{~b}$ & $5.61 \pm 1.02 \mathrm{~b}$ & $2.77 \pm 0.55 \mathrm{~b}$ & $3.96 \pm 0.70 \mathrm{~b}$ & $14.641 * * *$ & $6.196^{* * *}$ & \\
\hline & 2012 & Control & $0.189 \pm 0.050 \mathrm{~b}$ & $0.449 \pm 0.053 \mathrm{~b}$ & $2.31 \pm 0.33 \mathrm{a}$ & $0.224 \pm 0.106 b$ & $0.729 \pm 0.393 b$ & $0.612 \pm 0.183 \mathrm{~b}$ & $12.017 * * *$ & & $49.083^{* * *}$ \\
\hline & & Lso+ & $17.5 \pm 4.1 \mathrm{a}$ & $4.05 \pm 0.42 \mathrm{~b}$ & $8.71 \pm 1.03 \mathrm{~b}$ & $5.77 \pm 1.39 \mathrm{~b}$ & $4.15 \pm 0.31 \mathrm{~b}$ & $4.03 \pm 0.86 \mathrm{~b}$ & $8.040 * * *$ & & \\
\hline & 2013 & Control & $0.314 \pm 0.057$ & $0.284 \pm 0.032$ & $0.357 \pm 0.072$ & $0.261 \pm 0.054$ & $0.465 \pm 0.038$ & $0.365 \pm 0.110$ & 1.229 & & $46.600^{* * *}$ \\
\hline & & Lso+ & $5.71 \pm 1.12 \mathrm{a}$ & $3.02 \pm 0.89 \mathrm{ab}$ & $1.24 \pm 0.20 \mathrm{ab}$ & $5.32 \pm 1.57 \mathrm{a}$ & $0.271 \pm 0.040 \mathrm{~b}$ & $3.83 \pm 1.37 \mathrm{ab}$ & $4.410^{* * *}$ & & \\
\hline Flavonoid & & & & & & & & & & & \\
\hline glycoside 4 & Both & Control & $0.674 \pm 0.292 \mathrm{~b}$ & $1.16 \pm 0.24 \mathrm{ab}$ & $1.95 \pm 0.61 \mathrm{a}$ & $0.939 \pm 0.159 \mathrm{~b}$ & $0.470 \pm 0.119 \mathrm{~b}$ & $0.604 \pm 0.155 \mathrm{~b}$ & $4.802 * *$ & $37.871 * * *$ & $32.348 * * *$ \\
\hline & & Lso+ & $59.8 \pm 15.8 \mathrm{a}$ & $12.2 \pm 1.88 \mathrm{~b}$ & $12.8 \pm 2.6 \mathrm{~b}$ & $13.6 \pm 2.7 \mathrm{~b}$ & $5.72 \pm 1.28 \mathrm{~b}$ & $6.57 \pm 1.30 \mathrm{~b}$ & $8.706^{* * * *}$ & $17.993 * * *$ & \\
\hline & 2012 & Control & $1.19 \pm 0.44 \mathrm{~b}$ & $1.79 \pm 0.19 a b$ & $3.20 \pm 0.83 \mathrm{a}$ & $1.11 \pm 0.19 \mathrm{~b}$ & $0.715 \pm 0.155 \mathrm{~b}$ & $0.984 \pm 0.154 \mathrm{~b}$ & $4.919 * *$ & & $32.589^{* * *}$ \\
\hline & & Lso+ & $86.2 \pm 19.6 \mathrm{a}$ & $12.3 \pm 2.8 \mathrm{~b}$ & $18.7 \pm 2.4 \mathrm{~b}$ & $18.3 \pm 3.2 \mathrm{~b}$ & $8.84 \pm 0.85 \mathrm{~b}$ & $8.61 \pm 1.62 \mathrm{~b}$ & $13.135^{* * *}$ & & \\
\hline & 2013 & Control & $0.0534 \pm 0.0238 \mathrm{~b}$ & $0.412 \pm 0.072 \mathrm{ab}$ & $0.460 \pm 0.139 a \mathrm{ab}$ & $0.733 \pm 0.256 \mathrm{a}$ & $0.175 \pm 0.045 \mathrm{~b}$ & $0.147 \pm 0.038 \mathrm{~b}$ & $4.091 * *$ & & $118.960^{* * * *}$ \\
\hline & & Lso+ & $12.3 \pm 1.9 \mathrm{a}$ & $12.1 \pm 1.9 \mathrm{a}$ & $2.37 \pm 0.45 \mathrm{~b}$ & $5.05 \pm 0.92 \mathrm{~b}$ & $0.117 \pm 0.033 \mathrm{~b}$ & $2.91 \pm 0.866 \mathrm{~b}$ & $18.001^{* * * *}$ & & \\
\hline Flavonoid methyl & & & & & & & & & & & \\
\hline ester glycoside & Both & Control & $0.571 \pm 0.097 \mathrm{c}$ & $4.10 \pm 1.06 \mathrm{ab}$ & $5.12 \pm 1.40 \mathrm{a}$ & $2.58 \pm 0.64 \mathrm{bc}$ & $2.56 \pm 0.54 \mathrm{bc}$ & $1.69 \pm 0.35 \mathrm{c}$ & $7.815^{* * *}$ & $53.349 * * *$ & $62.362 * * *$ \\
\hline & & Lso+ & $28.4 \pm 3.6 \mathrm{a}$ & $22.1 \pm 5.2 \mathrm{a}$ & $29.7 \pm 7.0 \mathrm{a}$ & $4.65 \pm 0.68 \mathrm{~b}$ & $6.97 \pm 1.64 \mathrm{~b}$ & $3.01 \pm 0.45 \mathrm{~b}$ & $13.050^{* * * *}$ & $30.487 * * *$ & \\
\hline & 2012 & Control & $0.706 \pm 0.156 \mathrm{c}$ & $6.57 \pm 1.17 \mathrm{ab}$ & $8.21 \pm 1.71 \mathrm{a}$ & $4.02 \pm 0.75 \mathrm{bc}$ & $3.47 \pm 0.79 \mathrm{bc}$ & $2.53 \pm 0.37 \mathrm{bc}$ & $7.924^{* * * *}$ & & $57.295^{* * *}$ \\
\hline & & Lso+ & $28.5 \pm 4.5 \mathrm{a}$ & $31.0 \pm 6.3 \mathrm{a}$ & $44.5 \pm 6.8 \mathrm{a}$ & $6.04 \pm 0.69 \mathrm{~b}$ & $10.4 \pm 1.6 \mathrm{~b}$ & $3.96 \pm 0.42 b$ & $14.722^{* * * *}$ & & \\
\hline & 2013 & Control & $0.409 \pm 0.059 \mathrm{~b}$ & $1.13 \pm 0.30 \mathrm{ab}$ & $1.42 \pm 0.32 \mathrm{ab}$ & $0.843 \pm 0.050 \mathrm{ab}$ & $1.46 \pm 0.35 \mathrm{a}$ & $0.686 \pm 0.122 \mathrm{ab}$ & $3.153^{*}$ & & $26.179 * * *$ \\
\hline & & Lso+ & $28.1 \pm 6.8 \mathrm{a}$ & $5.89 \pm 0.94 \mathrm{~b}$ & $3.02 \pm 0.29 \mathrm{~b}$ & $2.14 \pm 0.27 \mathrm{~b}$ & $0.723 \pm 0.104 b$ & $1.29 \pm 0.28 \mathrm{~b}$ & $14.244^{* * *}$ & & \\
\hline Protocatechuic & & & & & & & & & & & \\
\hline acid glycoside & Both & Control & $14.0 \pm 7.4 \mathrm{~b}$ & $11.4 \pm 1.9 \mathrm{~b}$ & $6.93 \pm 2.31 \mathrm{~b}$ & $43.9 \pm 10.0 \mathrm{a}$ & $21.6 \pm 4.4 \mathrm{~b}$ & $17.9 \pm 6.0 \mathrm{~b}$ & $6.033^{* * * *}$ & $19.619^{* * * *}$ & $47.276^{* * * *}$ \\
\hline & & Lso+ & $78.9 \pm 7.1 \mathrm{a}$ & $29.3 \pm 4.3 \mathrm{~b}$ & $22.8 \pm 3.8 \mathrm{~b}$ & $60.3 \pm 7.5 \mathrm{a}$ & $26.2 \pm 6.9 \mathrm{~b}$ & $21.3 \pm 2.6 \mathrm{~b}$ & $21.759^{* * * *}$ & $12.308 * * *$ & \\
\hline & 2012 & Control & $23.4 \pm 12.7 \mathrm{~b}$ & $13.2 \pm 2.2 \mathrm{~b}$ & $6.19 \pm 4.0 \mathrm{~b}$ & $64.1 \pm 13.6 \mathrm{a}$ & $33.4 \pm 2.2 \mathrm{ab}$ & $25.1 \pm 10.2 \mathrm{~b}$ & $5.162 * *$ & & $14.489 * * *$ \\
\hline & & Lso+ & $90.5 \pm 8.6 \mathrm{a}$ & $20.0 \pm 3.9 \mathrm{~b}$ & $21.2 \pm 6.0 \mathrm{~b}$ & $74.4 \pm 8.4 \mathrm{a}$ & $39.1 \pm 7.8 \mathrm{~b}$ & $22.6 \pm 3.8 \mathrm{~b}$ & $20.672 * * *$ & & \\
\hline & 2013 & Control & $2.58 \pm 0.27 \mathrm{~b}$ & $9.30 \pm 3.22 b$ & $7.82 \pm 2.27 \mathrm{~b}$ & $19.6 \pm 1.6 \mathrm{a}$ & $7.55 \pm 2.43 b$ & $9.27 \pm 2.47 \mathrm{~b}$ & $6.168^{* * *}$ & & $197.335^{* * * *}$ \\
\hline & & Lso+ & $58.1 \pm 4.9 \mathrm{a}$ & $46.1 \pm 2.6 \mathrm{ab}$ & $25.7 \pm 1.1 \mathrm{~cd}$ & $35.0 \pm 3.6 \mathrm{bc}$ & $2.79 \pm 0.73 \mathrm{e}$ & $18.9 \pm 3.1 \mathrm{~d}$ & $41.777 * * *$ & & \\
\hline Quinic acid & & & & & & & & & & & \\
\hline derivative & Both & Control & $3.74 \pm 1.40 \mathrm{~b}$ & $1.63 \pm 0.11 \mathrm{~b}$ & $1.95 \pm 0.31 \mathrm{~b}$ & $12.9 \pm 3.9 \mathrm{a}$ & $3.24 \pm 0.35 \mathrm{~b}$ & $2.20 \pm 0.32 \mathrm{~b}$ & $14.245^{* * * *}$ & $21.564 * * *$ & $55.438 * * *$ \\
\hline & & Lso+ & $33.2 \pm 6.7 \mathrm{a}$ & $2.40 \pm 0.26 \mathrm{c}$ & $2.71 \pm 0.24 \mathrm{c}$ & $21.2 \pm 4.6 \mathrm{~b}$ & $7.17 \pm 0.83 \mathrm{c}$ & $3.48 \pm 0.41 \mathrm{c}$ & $43.936 * * *$ & $115.526 * * *$ & \\
\hline & 2012 & Control & $5.22 \pm 2.50 \mathrm{~b}$ & $1.69 \pm 0.13 b$ & $1.08 \pm 0.10 \mathrm{~b}$ & $22.8 \pm 3.7 \mathrm{a}$ & $2.85 \pm 0.32 \mathrm{~b}$ & $2.72 \pm 0.44 b$ & $20.165^{* * * *}$ & & $68.010^{* * * *}$ \\
\hline & & Lso+ & $50.1 \pm 3.6 \mathrm{a}$ & $2.99 \pm 0.21 \mathrm{c}$ & $2.57 \pm 0.36 \mathrm{c}$ & $32.0 \pm 3.6 \mathrm{~b}$ & $7.35 \pm 1.29 \mathrm{c}$ & $3.02 \pm 0.40 \mathrm{c}$ & $86.728^{* * * *}$ & & \\
\hline & 2013 & Control & $1.97 \pm 0.09 \mathrm{bc}$ & $1.55 \pm 0.19 \mathrm{c}$ & $2.99 \pm 0.15 a b$ & $1.03 \pm 0.14 \mathrm{c}$ & $3.72 \pm 0.65 \mathrm{a}$ & $1.57 \pm 0.32 \mathrm{c}$ & $10.149^{* * * *}$ & & $27.822 * * *$ \\
\hline & & Lso+ & $2.92 \pm 0.44 \mathrm{bc}$ & $1.34 \pm 0.11 \mathrm{c}$ & $2.96 \pm 0.21 b c$ & $1.70 \pm 0.29 \mathrm{c}$ & $6.85 \pm 0.48 \mathrm{a}$ & $4.32 \pm 0.85 b$ & $18.959^{* * * *}$ & & \\
\hline
\end{tabular}

${ }^{\mathrm{z} F}$ statistics are provided. Different letters represent significant mean separations by Tukey's honest significant difference test. $* P<0.05 ; * * P<0.01$; and $* * * P<0.001$. 
Regarding tuber levels of metabolites in Lso-inoculated plants, many more differences were observed between entries for both years of this study. This was important because changes in host chemistry have been consistently linked to ZC symptom development in tubers. This study examined shifts in amino acids, sugars, and phenolics that occurred after being infected with Lso for a substantial period (Rashed et al. 2013; Wallis et al. 2014). Positive correlations were indeed observed between the levels of many of these compounds with observed symptoms, again confirming previous reports that shifts in host chemistry were associated with ZC progression (Rashed et al. 2013).

For the majority of compounds in both years of this study, infected Atlantic had greater levels than any other entry. This was despite that, in many cases, healthy tubers of Atlantic did not initially chemically differ from those of tubers of breeding clones. Especially apparent was that when comparing fold increases in compound levels of infected tuber levels compared with noninfected tubers, Atlantic tubers generally underwent far greater increases when infected compared with any other of the entries. Since most compounds were positively correlated with symptoms, it therefore makes since that such large increases in compound levels of Atlantic tubers following Lso infection resulted in very apparent ZC symptoms, whereas in the other entries symptoms were missing or less apparent. Since a very clear induction of many analyzed compounds occurred in Atlantic versus other entries following Lso infection, the possibility exists that transcripts or proteins might be discovered that reduce host responses to Lso, which in turn could be useful resistance markers for breeding programs. Such hypothetical proteins may be expressed on the surface (with leucine-rich repeat domains) and suppress or block host recognition of the pathogen instead of triggering a host response. This study therefore suggests that if an Lso tolerance marker is found through genomic analyses it should be screened to see if it has putative host physiology regulatory functions in additional to suppression of pathogen growth.

It should be noted that this study, due to the complexities of chemical analyses as well as a limited amount of field space for repeated screenings, omitted breeding lines exhibiting no to light ZC symptoms when freshly cut, which was an additional 24 lines compared with the five selected for analyses here. The study also did not include breeding lines exhibiting moderate and not severe symptoms. Inclusion of these additional lines should be considered in future studies as these could provide further support that a gradient of host physiological responses results in a gradient of ZC symptom development. In addition, physiological responses of one

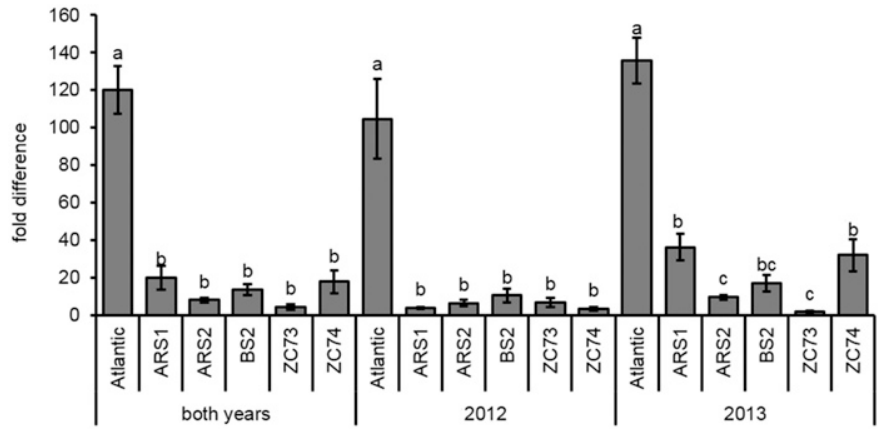

Fig. 6. Fold differences of phenolic compound levels for 'Candidatus Liberibacter solanacearum'-infected tubers over control tubers of each entry for both years and each year separately. Bars represent standard errors. Different letters represent means that are significantly different by Tukey's honest significant difference test.

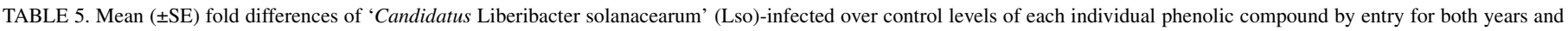
each year separately ${ }^{\mathrm{z}}$

\begin{tabular}{|c|c|c|c|c|c|c|c|c|c|}
\hline \multirow[b]{2}{*}{ Compound } & \multirow[b]{2}{*}{ Year } & \multirow[b]{2}{*}{ Atlantic } & \multirow[b]{2}{*}{ ARS1 } & \multirow[b]{2}{*}{ ARS2 } & \multirow[b]{2}{*}{ BS2 } & \multirow[b]{2}{*}{ ZC73 } & \multirow[b]{2}{*}{$\mathrm{ZC74}$} & \multicolumn{2}{|c|}{$F$} \\
\hline & & & & & & & & $\begin{array}{c}\text { Between } \\
\text { entry }\end{array}$ & $\begin{array}{c}\text { Between } \\
\text { year }\end{array}$ \\
\hline \multirow[t]{3}{*}{ Chlorogenic acid } & Both & $191.39 \pm 86.37 \mathrm{a}$ & $15.16 \pm 3.80 \mathrm{~b}$ & $6.66 \pm 1.03 \mathrm{~b}$ & $8.17 \pm 2.13 b$ & $3.37 \pm 0.83 b$ & $10.03 \pm 3.74 \mathrm{~b}$ & $5.513 * * *$ & 2.302 \\
\hline & 2012 & $328.18 \pm 155.26 \mathrm{a}$ & $5.10 \pm 1.80 \mathrm{~b}$ & $6.88 \pm 1.63 b$ & $7.71 \pm 3.89 \mathrm{~b}$ & $3.50 \pm 0.78 b$ & $1.66 \pm 0.76 \mathrm{~b}$ & $4.330^{* *}$ & \\
\hline & 2013 & $54.61 \pm 10.33 \mathrm{a}$ & $25.22 \pm 3.37 \mathrm{~b}$ & $6.45 \pm 1.44 \mathrm{~cd}$ & $8.64 \pm 2.28 \mathrm{~cd}$ & $3.23 \pm 1.58 \mathrm{~d}$ & $18.41 \pm 5.23 \mathrm{bc}$ & $14.056^{* * *}$ & \\
\hline \multirow[t]{3}{*}{ Chlorogenic acid derivative } & Both & $128.62 \pm 58.34 \mathrm{a}$ & $27.74 \pm 9.47 b$ & $3.67 \pm 0.61 \mathrm{~b}$ & $5.20 \pm 0.93 \mathrm{~b}$ & $4.30 \pm 1.15 b$ & $24.19 \pm 16.01 \mathrm{~b}$ & $4.544^{* *}$ & $5.957 *$ \\
\hline & 2012 & $39.78 \pm 5.47 \mathrm{a}$ & $2.49 \pm 0.76 b$ & $3.34 \pm 0.55 b$ & $4.15 \pm 1.05 \mathrm{~b}$ & $6.59 \pm 1.73 b$ & $1.68 \pm 0.29 \mathrm{~b}$ & $37.870^{* * * *}$ & \\
\hline & 2013 & $217.45 \pm 106.48 \mathrm{a}$ & $52.99 \pm 9.18 \mathrm{~b}$ & $4.00 \pm 1.14 \mathrm{~b}$ & $6.25 \pm 1.50 \mathrm{~b}$ & $2.00 \pm 0.59 \mathrm{~b}$ & $46.70 \pm 30.01 \mathrm{~b}$ & $3.334^{*}$ & \\
\hline \multirow[t]{3}{*}{ Cryptochlorogenic acid } & Both & $124.75 \pm 19.37 \mathrm{a}$ & $21.37 \pm 6.63 \mathrm{~b}$ & $6.55 \pm 1.09 \mathrm{~b}$ & $9.81 \pm 2.06 \mathrm{~b}$ & $3.72 \pm 1.01 \mathrm{~b}$ & $54.31 \pm 35.72 b$ & $8.623 * * *$ & 3.444 \\
\hline & 2012 & $122.31 \pm 28.92 a$ & $2.83 \pm 0.83 \mathrm{~b}$ & $7.06 \pm 2.05 \mathrm{~b}$ & $7.43 \pm 2.98 b$ & $5.72 \pm 1.55 b$ & $2.48 \pm 0.52 \mathrm{~b}$ & $16.135^{* * * *}$ & \\
\hline & 2013 & $127.19 \pm 29.14 \mathrm{a}$ & $39.92 \pm 5.05 \mathrm{bc}$ & $6.03 \pm 0.99 \mathrm{c}$ & $12.19 \pm 2.71 \mathrm{c}$ & $1.72 \pm 0.43 \mathrm{c}$ & $106.13 \pm 66.31 \mathrm{ab}$ & $3.386^{*}$ & \\
\hline \multirow[t]{3}{*}{ Cryptochlorogenic acid derivative } & Both & $103.80 \pm 39.22 \mathrm{a}$ & $21.79 \pm 9.60 \mathrm{~b}$ & $19.11 \pm 6.23 \mathrm{~b}$ & $50.06 \pm 18.19 \mathrm{ab}$ & $4.92 \pm 3.21 \mathrm{~b}$ & $12.19 \pm 6.31 \mathrm{~b}$ & $6.652 * * *$ & 0.182 \\
\hline & 2012 & $178.60 \pm 62.83 \mathrm{a}$ & $1.07 \pm 0.53 \mathrm{~b}$ & $3.87 \pm 1.38 \mathrm{~b}$ & $2.94 \pm 1.28 \mathrm{~b}$ & $9.46 \pm 6.00 \mathrm{~b}$ & $0.97 \pm 0.30 \mathrm{~b}$ & $7.690 * * *$ & \\
\hline & 2013 & $29.00 \pm 13.37 \mathrm{bc}$ & $42.51 \pm 14.12 \mathrm{ab}$ & $34.36 \pm 7.51 \mathrm{bc}$ & $97.17 \pm 19.43 \mathrm{a}$ & $0.37 \pm 0.09 \mathrm{c}$ & $23.41 \pm 10.78 \mathrm{bc}$ & $6.780 * * *$ & \\
\hline \multirow[t]{3}{*}{ Flavonoid glycoside 1} & Both & $28.02 \pm 9.92 b$ & $101.64 \pm 46.43 \mathrm{a}$ & $28.61 \pm 11.06 \mathrm{~b}$ & $11.11 \pm 4.78 \mathrm{~b}$ & $3.20 \pm 2.10 \mathrm{~b}$ & $13.01 \pm 8.03 b$ & $5.520 * * *$ & $19.806^{* * * *}$ \\
\hline & 2012 & $5.13 \pm 1.00 \mathrm{a}$ & $3.33 \pm 0.77 \mathrm{ab}$ & $7.00 \pm 2.84 \mathrm{a}$ & $0.95 \pm 0.28 \mathrm{~b}$ & $1.11 \pm 0.28 b$ & $0.83 \pm 0.39 \mathrm{~b}$ & $3.982 * *$ & \\
\hline & 2013 & $50.90 \pm 13.40 \mathrm{~b}$ & $199.95 \pm 69.76 \mathrm{a}$ & $50.23 \pm 17.58 b$ & $21.27 \pm 7.14 \mathrm{~b}$ & $5.29 \pm 4.19 b$ & $25.19 \pm 14.69 \mathrm{~b}$ & $5.418 * *$ & \\
\hline \multirow[t]{3}{*}{ Flavonoid glycoside 2} & Both & $93.94 \pm 20.68 \mathrm{a}$ & $9.74 \pm 4.24 b$ & $3.36 \pm 0.85 b$ & $33.15 \pm 20.10 b$ & $1.59 \pm 0.35 b$ & $10.17 \pm 5.73 \mathrm{~b}$ & $7.891^{* * *}$ & 0.436 \\
\hline & 2012 & $98.17 \pm 35.81 \mathrm{a}$ & $4.07 \pm 1.55 \mathrm{~b}$ & $4.00 \pm 1.53 \mathrm{~b}$ & $47.36 \pm 40.06 \mathrm{ab}$ & $1.75 \pm 0.51 b$ & $17.01 \pm 11.11 \mathrm{~b}$ & $2.887^{*}$ & \\
\hline & 2013 & $89.71 \pm 25.16 \mathrm{a}$ & $15.42 \pm 7.89 \mathrm{~b}$ & $2.71 \pm 0.85 b$ & $18.94 \pm 10.58 b$ & $1.44 \pm 0.53 b$ & $3.32 \pm 0.91 \mathrm{~b}$ & $8.572 * * *$ & \\
\hline \multirow[t]{3}{*}{ Flavonoid glycoside 3} & Both & $54.17 \pm 23.56 \mathrm{a}$ & $9.03 \pm 1.23 \mathrm{~b}$ & $4.02 \pm 0.88 \mathrm{~b}$ & $31.97 \pm 6.99 \mathrm{ab}$ & $6.54 \pm 3.17 b$ & $12.02 \pm 4.76 \mathrm{~b}$ & $4.038 * *$ & 3.133 \\
\hline & 2012 & $87.36 \pm 43.81 \mathrm{a}$ & $7.92 \pm 0.88 \mathrm{~b}$ & $3.27 \pm 0.39 \mathrm{~b}$ & $36.83 \pm 7.75 \mathrm{ab}$ & $12.46 \pm 5.25 \mathrm{~b}$ & $12.34 \pm 9.39 \mathrm{~b}$ & $2.916^{*}$ & \\
\hline & 2013 & $20.98 \pm 5.36 \mathrm{ab}$ & $10.15 \pm 2.32 \mathrm{bc}$ & $4.78 \pm 1.74 \mathrm{bc}$ & $27.12 \pm 12.16 \mathrm{a}$ & $0.62 \pm 0.14 \mathrm{c}$ & $11.69 \pm 3.71 \mathrm{abc}$ & $2.971^{*}$ & \\
\hline \multirow[t]{3}{*}{ Flavonoid glycoside 4} & Both & $429.03 \pm 215.07 \mathrm{a}$ & $19.23 \pm 4.82 \mathrm{~b}$ & $10.40 \pm 4.45 \mathrm{~b}$ & $17.91 \pm 4.78 \mathrm{~b}$ & $8.17 \pm 2.69 \mathrm{~b}$ & $27.28 \pm 15.87 \mathrm{~b}$ & $4.844 * *$ & $4.220 *$ \\
\hline & 2012 & $58.71 \pm 12.87 \mathrm{a}$ & $6.65 \pm 0.57 \mathrm{~b}$ & $14.62 \pm 8.83 b$ & $24.34 \pm 7.83 b$ & $15.41 \pm 2.51 \mathrm{~b}$ & $6.99 \pm 1.24 \mathrm{~b}$ & $7.305 * * *$ & \\
\hline & 2013 & $799.36 \pm 373.38 \mathrm{a}$ & $31.80 \pm 5.01 \mathrm{~b}$ & $6.18 \pm 1.45 \mathrm{~b}$ & $11.48 \pm 4.57 \mathrm{~b}$ & $0.93 \pm 0.30 \mathrm{~b}$ & $47.56 \pm 30.44 \mathrm{~b}$ & $4.344 * *$ & \\
\hline \multirow{4}{*}{$\begin{array}{l}\text { Flavonoid methyl ester } \\
\text { glycoside }\end{array}$} & & & & & & & & & \\
\hline & Both & $54.35 \pm 14.76 \mathrm{a}$ & $6.15 \pm 1.78 b$ & $4.90 \pm 1.34 b$ & $2.39 \pm 0.31 b$ & $2.49 \pm 0.99 b$ & $1.75 \pm 0.32 \mathrm{~b}$ & $14.535^{* * * *}$ & 2.673 \\
\hline & 2012 & $30.45 \pm 4.75 \mathrm{a}$ & $4.46 \pm 1.23 \mathrm{~b}$ & $7.42 \pm 2.18 b$ & $2.17 \pm 0.48 \mathrm{~b}$ & $4.31 \pm 1.66 \mathrm{~b}$ & $1.36 \pm 0.18 b$ & $22.914 * * *$ & \\
\hline & 2013 & $78.26 \pm 25.92 \mathrm{a}$ & $7.84 \pm 3.35 \mathrm{~b}$ & $2.38 \pm 0.34 \mathrm{~b}$ & $2.62 \pm 0.42 b$ & $0.67 \pm 0.19 b$ & $2.14 \pm 0.59 \mathrm{~b}$ & $8.310^{* * *}$ & \\
\hline \multirow[t]{3}{*}{ Protocatechuic acid glycoside } & Both & $14.86 \pm 3.74 \mathrm{a}$ & $4.84 \pm 1.37 \mathrm{~b}$ & $6.38 \pm 1.83 \mathrm{~b}$ & $1.53 \pm 0.26 \mathrm{~b}$ & $0.94 \pm 0.29 \mathrm{~b}$ & $2.06 \pm 0.57 \mathrm{~b}$ & $16.045^{* * *}$ & $15.079 * * *$ \\
\hline & 2012 & $5.52 \pm 1.32 \mathrm{ab}$ & $2.18 \pm 0.51 \mathrm{bc}$ & $7.13 \pm 3.23 \mathrm{a}$ & $1.16 \pm 0.32 \mathrm{c}$ & $1.01 \pm 0.26 \mathrm{c}$ & $1.22 \pm 0.58 \mathrm{c}$ & $3.202 *$ & \\
\hline & 2013 & $24.20 \pm 4.20 \mathrm{a}$ & $7.49 \pm 2.15 \mathrm{bc}$ & $5.64 \pm 2.09 \mathrm{c}$ & $1.90 \pm 0.35 \mathrm{bc}$ & $0.87 \pm 0.56 \mathrm{c}$ & $2.91 \pm 0.88 \mathrm{bc}$ & $16.313^{* * * *}$ & \\
\hline \multirow[t]{3}{*}{ Quinic acid derivative } & Both & $9.92 \pm 3.95 \mathrm{a}$ & $1.38 \pm 0.20 \mathrm{~b}$ & $1.58 \pm 0.31 \mathrm{~b}$ & $1.55 \pm 0.25 \mathrm{~b}$ & $2.12 \pm 0.28 \mathrm{~b}$ & $2.11 \pm 0.61 \mathrm{~b}$ & $7.347 * * *$ & $7.222 * *$ \\
\hline & 2012 & $18.33 \pm 5.89 \mathrm{a}$ & $1.85 \pm 0.24 \mathrm{~b}$ & $2.14 \pm 0.51 \mathrm{~b}$ & $1.31 \pm 0.34 \mathrm{~b}$ & $2.23 \pm 0.53 \mathrm{~b}$ & $0.91 \pm 0.16 \mathrm{~b}$ & $7.855^{* * *}$ & \\
\hline & 2013 & $1.50 \pm 0.23 \mathrm{~b}$ & $0.91 \pm 0.13 \mathrm{~b}$ & $1.01 \pm 0.12 \mathrm{~b}$ & $1.78 \pm 0.36 \mathrm{~b}$ & $2.00 \pm 0.26 \mathrm{~b}$ & $3.31 \pm 0.96 \mathrm{a}$ & $3.837 *$ & \\
\hline
\end{tabular}

$\mathrm{z}$ Values over 1 represent greater levels in Lso-infected tubers, whereas values under 1 represent levels greater in noninfected control. $F$ statistics are provided. Different letters represent significant mean separations by Tukey's honest significant difference test. $* P<0.05 ; * * P<0.01$; and $* * * P<0.001$. 
type of compound, such as phenolics, may be distinct from those of other types, such as sugars and amino acids, leading to the reduction of some visible symptoms (such as fresh symptoms) but not others (such as fried symptoms).

Results from this study support a hypothesis that Liberibacter bacteria such as Lso may exist as non-disease-causing endophytic parasites in wild host species. With the expansion of commercial agriculture, these bacteria were able to parasitize commercial cultivars of plants that displayed increased sensitivity to infections and therefore greater symptom development, likely because when commercial cultivars were developed traits beneficial to commercial success were selected and not traits providing increased resistance to unforeseen parasites. If true, larger-scale studies aimed at purposely identifying and characterizing endophytic bacteria and other microorganisms appear warranted in wild plant relatives of many commercial crops, as these might eventually become important emerging pathogens in the future when finally given access to susceptible hosts, efficient vectors, or if they are moved into new areas.

These results also hypothetically could be applied to additional Liberibacter-caused diseases, such as citrus greening (huanglongbing, caused by 'Candidatus Liberibacter asiaticum', 'Candidatus Liberibacter americanus', or 'Candidatus Liberibacter africanum') or recent Liberibacter-caused diseases of carrots and celery in Europe (with European strains of Lso as putative causal agents) (Munyaneza 2012). For those diseases, it remains relatively unknown what changes in host physiology occur throughout the infection process. However, it is plausible that because Liberibacter bacteria live an endophytic lifestyle within plant hosts, they likely evolved in wild host species and, as a result, germplasm from such sources may provide tolerance in the form of milder host responses to infection.

In conclusion, this study demonstrated that Lso tolerance in potato germplasm is present and is characterized by reduced Lso titers or even changes in tuber biochemistry relative to susceptible potato cultivars such as Atlantic. This potato germplasm as well as other wild solanaceous species-derived germplasm can be used in developing new potato varieties for those potato growing regions where ZC is a major concern. This study also observed that a lack of biochemical changes upon Lso infection might be a potential mechanism for ZC resistance in addition to reductions in Lso progression, and this trait can be screened for in future trials to develop new varieties. Alleles that control the intensity of host response to Lso could eventually be decoded and used as genetic markers of $\mathrm{ZC}$ resistance to speed selections and ultimately mitigate the threat $\mathrm{ZC}$ disease poses to potato production everywhere.

\section{ACKNOWLEDGMENTS}

We thank B. Heilman, V. Sengoda, M. Heidt, F. de la Rosa, J. Dixon, S. Delp, J. Gefre, M. Henning, G. Aguilar, A. Fite, N. Goodell, A. Wallingford, N. Fonseca, and J. Pedraza for their technical help in this work. Special thanks to S. Jansky, USDA-ARS, Madison, WI, from the ARS clones used in this study. The USDA-NIFA-Specialty Crop Research Initiative (grant 2009-51181-20176) and associated Zebra Chip SCRI/Industry-Funded Mini-Grant Program, USDA-NIFA-RAMP (Project 2009-51101-05892), Frito-Lay Inc., and Texas Department of Agriculture funded this research. Mention of trade names or commercial products in this publication is solely for the purpose of providing specific information and does not imply recommendation or endorsement by the U.S. Department of Agriculture. USDA is an equal opportunity provider and employer.

\section{LITERATURE CITED}

Alvarado, V. Y., Odokonyero, D., Duncan, O., Mirkov, T. E., and Scholthof, H. B. 2012. Molecular and physiological properties associated with zebra chip complex disease in potatoes and its relation with Candidatus Liberibacter contents in psyllid vectors. PLoS One 7:e37345.

Buchman, J. L., Fisher, T. W., Sengoda, V. G., and Munyaneza, J. E. 2012. Zebra chip progression: From inoculation of potato plants with Liberibacter to development of disease symptoms in tubers. Am. J. Potato Res. 89: 159-168.

Buchman, J. L., Heilman, B. E., and Munyaneza, J. E. 2011a. Effects of Bactericera cockerelli (Hemiptera: Triozidae) density on zebra chip potato disease incidence, potato yield, and tuber processing quality. J. Econ. Entomol. 104:1783-1792.

Buchman, J. L., Sengoda, V. G., and Munyaneza, J. E. 2011b. Vector transmission efficiency of Liberibacter by Bactericera cockerelli (Hemiptera: Triozidae) in zebra chip potato disease: Effects of psyllid life stage and inoculation access period. J. Econ. Entomol. 104:1486-1495.

Butler, C. D., Gonzalez, B., Manjunath, K., Lee, R. F., Novy, R. G., Miller, C., Jr., and Trumble, J. T. 2011. Behavioral responses of adult potato psyllid, Bactericera cockerelli (Hemiptera: Triozidae), to potato germplasm and transmission of Candidatus Liberibacter psyllaurous. Crop Prot. 30:1233-1238.

Cooper, W. R., and Bamberg, J. B. 2014. Variation in Bactericera cockerelli (Hemipter: Triozidae) oviposition, survival, and development on Solanum bulbocastanum germplasm. Am. J. Potato Res. 91:532-537.

Crosslin, J. M., Hamm, P. B., Eggers, J. E., Rondon, S. I., Sengoda, V. G., and Munyaneza, J. E. 2012a. First report of zebra chip disease and "Candidatus Liberibacter solanacearum" on potatoes in Oregon and Washington State. Plant Dis. 96:452.

Crosslin, J. M., Olsen, N., and Nolte, P. 2012b. First report of zebra chip disease and "Candidatus Liberibacter solanacearum" on potatoes in Idaho. Plant Dis. 96:453.

Diaz-Montano, J., Vindiola, B. G., Drew, N., Novy, R. G., Miller, J. C., and Trumble, J. T. 2014. Resistance of selected potato genotypes to the potato psyllid (Hemiptera: Triozidae). Am. J. Potato Res. 91:363-367.

Friedman, M., and Levin, C. E. 2008. Review of methods for the reduction of dietary content and toxicity of acrylamide. J. Agric. Food Chem. 56: 6113-6140.

Greenway, G. 2014. Economic impact of zebra chip control costs on grower returns in seven US states. Am. J. Potato Res. 91:714-719.

Guenthner, J., Goolsby, J., and Greenway, G. 2012. Use and cost of insecticides to control potato psyllids and zebra chip on potatoes. Southwest. Entomol. 37:263-270.

Henriquez, M. A., Adam, L. R., and Daayf, F. 2012. Alteration of secondary metabolites' profile in potato leaves in response to weakly and highly aggressive isolates of Phytophora infestans. Plant Physiol. Biochem. 57:8-14.

Jansky, S. H., Hamernik, A., and Bethke, P. C. 2011. Germplasm release: Tetraploid clones with resistance to cold-induced sweetening. Am. J. Potato Res. 88:218-225.

Levy, J., and Tamborindeguy, C. 2014. Solanum habrochaites, a potential source of resistance against Bactericera cockerelli (Hemiptera: Triozidae) and 'Candidatus Liberibacter solanacearum'. J. Econ. Entomol. 107: 1187-1193.

Liefting, L. W., Perez-Egusquiza, Z. C., Clover, G. R. G., and Anderson, J. A. D. 2008. A new 'Candidatus Liberibacter' species in Solanum tuberosum in New Zealand. Plant Dis. 92:1474.

Mayer, A. M. 2006. Polyphenol oxidases in plants and fungi: going places? A review. Phytochemistry 67:2318-2331.

Miles, G. P., Buchman, J. L., and Munyaneza, J. E. 2009. Impact of zebra chip disease on the mineral content of potato tubers. Am. J. Potato Res. 86: 481-489.

Miles, G. P., Samuel, M. A., Chen, J., Civerolo, E. L., and Munyaneza, J. E. 2010. Evidence that cell death is associated with zebra chip disease in potato tubers. Am. J. Potato Res. 87:337-349.

Munyaneza, J. E. 2012. Zebra chip disease of potato: Biology, epidemiology, and management. Am. J. Potato Res. 89:329-350.

Munyaneza, J. E., Buchman, J. L., Sengoda, V. G., Fisher, T. W., and Pearson, C. C. 2011. Susceptibility of selected potato varieties to zebra chip potato disease. Am. J. Potato Res. 88:435-440.

Munyaneza, J. E., Crosslin, J. M., and Upton, J. E. 2007. Association of Bactericera cockerelli (Homoptera: Psyllidae) with "zebra chip", a new potato disease in southwestern United States and Mexico. J. Econ. Entomol. 100:656-663.

Navarre, D. A., Shakya, R., Holden, J., and Crosslin, J. M. 2009. LC-MS analysis of phenolic compounds in tubers showing zebra chip symptoms. Am. J. Potato Res. 86:88-95.

Ngadze, E., Icishahayo, D., Coutinho, T. A., and van der Waals, J. E. 2012. Role of polyphenol oxidase, peroxidase, phenylalanine ammonia lyase, chlorogenic acid, and total soluble phenols in resistance of potatoes to soft rot. Plant Dis. 96:186-192.

Prager, S. M., Vindiola, B., Kund, G. S., Byrne, F. J., and Trumble, J. T. 2013. Considerations for the use of neonicotinoid pesticides in management of Bactericera cockerelli (Sulk) (Hemiptera: Triozidae). Crop Prot. 54:84-91.

Rashed, A., Wallis, C. M., Paetzold, L., Workneh, F., and Rush, C. M. 2013. Zebra chip disease and potato biochemistry: Tuber physiological changes in response to 'Candidatus Liberibacter solanacearum' infection over time. Phytopathology 103:419-426. 
Secor, G. A., and Rivera-Varas, V. 2004. Emerging diseases of cultivated potato and their impact on Latin America. Rev. Latinoam. Papa $1: 1-8$.

Secor, G. A., Rivera-Varas, V., Abad, J. A., Lee, I. M., Clover, G. R. G., Liefting, L. W., Li, X., and De Boer, S. H. 2009. Association of 'Candidatus Liberibacter solanacearum' with zebra chip disease of potato established by graft and psyllid transmission, electron microscopy, and PCR. Plant Dis. 93: 574-583.

Stadler, R. H., Blank, I., Varga, N., Robert, F., Hau, J., Guy, P. A., Robert, M.-C., and Riediker, S. 2002. Acrylamide from Maillard reaction products. Nature 419:449-450.

Wallis, C., Eyles, A., McSpadden Gardener, B., Hansen, R., Cipollini, D., Herms, D. A., and Bonello, P. 2008. Systemic induction of phloem secondary metabolism and its relationship to resistance to a canker pathogen in Austrian pine. New Phytol. 177:767-778.
Wallis, C. M., Chen, J., and Civerolo, E. L. 2012. Zebra chip-diseased potato tubers are characterized by increased levels of host phenolics, amino acids, and defense-related proteins. Physiol. Mol. Plant Pathol. 78:66-72.

Wallis, C. M., Rashed, A., Chen, J., Paetzold, L., Workneh, F., and Rush, C. M. 2015. Effects of potato psyllid-vectored 'Candidatus Liberibacter solanacearum' infection on potato leaf and stem physiology. Phytopathology 105:189-198.

Wallis, C. M., Rashed, A., Wallingford, A., Paetzold, L., Workneh, F., and Rush, C. M. 2014. Similarities and differences in physiological response to 'Candidatus Liberibacter solanacearum' infection among different potato cultivars. Phytopathology 104:126-133.

Wen, A., Mallik, I., Alvarado, V. Y., Pasche, J. S., Wang, X., Li, W., Levy, L., Lin, H., Scholthof, H. B., Mirkov, T. E., Rush, C. M., and Gudmestad, N. C. 2009. Detection, distribution, and genetic variability of 'Candidatus Liberibacter' species associated with zebra complex disease of potato in North America. Plant Dis. 93:1102-1115. 